\title{
Characterization of the disappearance and formation of biohydrogenation intermediates during incubations of linoleic acid with rumen fluid in vitro ${ }^{1}$
}

\author{
A. M. Honkanen, ${ }^{*}$ J. M. Griinari, $†$ A. Vanhatalo, ${ }^{*}$ S. Ahvenjärvi, ‡ V. Toivonen, $\neq$ and K. J. Shingfield ${ }^{2}$ \\ *Department of Agricultural Sciences, PO Box 28, FI-00014, University of Helsinki, Finland \\ †Department of Animal Nutrition and Management, The Swedish University of Agricultural Sciences, Kungsängen Research Centre, \\ SE-753 23 Uppsala, Sweden \\ ¥Animal Production Research, MTT Agrifood Research Finland, FI-31600, Jokioinen, Finland
}

\begin{abstract}
Dietary unsaturated fatty acids are extensively hydrogenated in the rumen, resulting in the formation of numerous intermediates that may exert physiological effects and alter the fat composition of ruminantderived foods. A batch culture method was used to characterize the hydrogenation of linoleic acid (LeA) by strained rumen fluid in vitro. Incubations $(\mathrm{n}=5)$ were performed in $100-\mathrm{mL}$ flasks maintained at $39^{\circ} \mathrm{C}$ containing $400 \mathrm{mg}$ of grass hay, $50 \mathrm{~mL}$ of buffered rumen fluid, and incremental amounts of LeA $(0,1.0,2.5$, 5.0 , or $10.0 \mathrm{mg}$ ) for $0,1.5,3.0,4.5,6.0$, and $9.0 \mathrm{~h}$. The fatty acid composition of flask contents was determined using complimentary silver-ion thin-layer chromatography, gas chromatography mass-spectrometry, and silver-ion high-performance liquid chromatography. Linoleic acid was extensively (98.1, 97.6, 98.0, and $89.8 \%$ for additions of $1.0,2.5,5.0$, and $10.0 \mathrm{mg}$ of LeA, respectively) hydrogenated over time. Complete reduction of LeA to 18:0 was inhibited in direct relation to the amount of added substrate, the extent of which was greatest for the highest amount of LeA addition. Recoveries of 1.0, 2.5, 5.0, and $10.0 \mathrm{mg}$ of added LeA as 18:0 averaged $73.6,65.0,57.3$, and $10.7 \%$, respectively. Incubation of incremental amounts of LeA resulted in a time-dependent accumulation of geometric isomers of 9,11 and 10,12 conjugated linoleic acid, several nonconjugated 18:2 isomers, and a wide range of cis 18:1 and trans 18:1 intermediates. Several unusual intermediates including cis-6,cis-12 18:2; cis-7,cis-12 18:2; and cis-8,cis-12 18:2, were found to accumulate in direct relation to the amount of added LeA, providing the first indications that hydrogenation of LeA by ruminal bacteria may also involve mechanisms other than hydrogen abstraction or isomerization of the cis-12 double
\end{abstract}

Received March 24, 2011.

Accepted October 18, 2011.

${ }^{1}$ Supported in part by the Raisio Science Foundation (Raisio, Finland).

${ }^{2}$ Corresponding author: kevin.shingfield@mtt.fi bond. Fitting of single-pool, first-order kinetic models to experimental data indicated that the rate of LeA disappearance decreased with increases in substrate availability. Reduction of $18: 1$ and 18:2 intermediates occurred at much lower rates compared with conjugated linoleic acid and nonconjugated 18:2 isomer formation. In conclusion, the extent of LeA biohydrogenation in vitro was shown to be time- and dose-dependent with evidence that LeA is hydrogenated by ruminal bacteria via several distinct metabolic pathways. The accumulation of several unusual 18:2 isomers indicates that biohydrogenation of LeA also proceeds via mechanisms other than isomerization of the cis-12 double bond.

Key words: biohydrogenation, linoleic acid, conjugated linoleic acid, trans fatty acid

\section{INTRODUCTION}

Characterizing the formation of specific biohydrogenation metabolites in the rumen is important with respect to understanding the metabolic origins of trans fatty acids in ruminant-derived foods and also the mechanisms underlying physiological responses to lipids in the diet of growing and lactating ruminants. On entering the rumen, dietary lipids are exposed to microbial lipases and the NEFA liberated are subject to biohydrogenation. Bacteria, rather than protozoa, are thought to be responsible, but relatively few strains capable of biohydrogenation have been identified (Harfoot and Hazlewood, 1988; Lourenço et al., 2010). Numerous in vitro and in vivo studies have allowed the major pathways of linoleic acid (LeA) biohydrogenation in the rumen to be elucidated (Harfoot and Hazlewood, 1988), but limited information is available on the formation of other intermediates (Troegeler-Meynadier et al., 2003; Jouany et al., 2007; Wallace et al., 2007).

Disappearance of LeA and formation of fatty acid intermediates over time during incubations with strained rumen fluid was determined to provide a detailed and comprehensive assessment of LeA metabolism in vitro. Intermediates formed during incubations with incremental amounts of LeA were identified and quantified 
using complimentary argentation silver-ion thin-layer chromatography $\left(\mathbf{A g}^{+}\right.$- TLC), silver-ion HPLC $\left(\mathbf{A g}^{+}\right.$HPLC), gas chromatography (GC), and GC-MS analysis of fatty acid methyl esters (FAME) and GCMS analysis of corresponding 4,4-dimethyloxazoline (DMOX) derivatives.

\section{MATERIALS AND METHODS}

\section{In Vitro Incubations}

Linoleic acid (cis-9,cis-12 18:2) obtained from a commercial source (Larodan Fine Chemicals AB, Malmö, Sweden) was incubated as an oil-in-water suspension (Rainio et al., 2001). Four stock solutions of LeA suspensions containing $0.4,1.0,2.0$, and $4.0 \mathrm{mg}$ of LeA/ $\mathrm{mL}$ were prepared daily, immediately before incubations with strained rumen fluid. Suspensions were prepared by mixing deionized water $(270,665,1,330$, and $2,660 \mu \mathrm{L})$ with Tween $80(10.0,25.0,50.0$, and $100 \mathrm{mg})$ and LeA $(8.0,20.0,40.0$, and $80.0 \mathrm{mg})$, resulting in a final LeA concentration of $0.4,1.0,2.0$, and $4.0 \mathrm{mg}$ of LeA/mL. Linoleic acid was dispersed by repeated flushing with a pipette, followed by the dropwise addition of $2 \mathrm{M} \mathrm{NaOH}(1,3,6$, and 12 drops, respectively) to each stock solution and gentle shaking until all solutions were clear. Once prepared, suspensions of LeA were transferred to $20-\mathrm{mL}$ volumetric flasks and diluted with deionized water. Amounts of LeA, Tween 80, and $\mathrm{NaOH}$ added to each flask were adjusted to be in same ratio for all incubations.

Rumen fluid was sampled from 1 nonlactating Finnish Ayrshire cow fitted with a rumen cannula and fed 8.7 $\mathrm{kg}$ of $\mathrm{DM} / \mathrm{d}$ of a diet (forage:concentrate ratio 70:30, on a DM basis) comprising timothy-meadow fescue silage supplemented with a standard commercial compound feed (Suomen Rehu Oy, Espoo, Finland), declared as containing 166, 76, and $40 \mathrm{~g} / \mathrm{kg}$ of $\mathrm{DM}$ of $\mathrm{CP}$, crude fiber, and ether extract, respectively, for 12 wk before sample collection.

One and a half liters of rumen fluid was collected from the donor cow using a vacuum pump $1 \mathrm{~h}$ after feeding and transported in a thermos flask to the in vitro laboratory within $10 \mathrm{~min}$. On arrival, rumen fluid was strained through 2 layers of cheesecloth to retain small particles, and mixed $(1: 2, \mathrm{vol} / \mathrm{vol})$ with warm $\left(39^{\circ} \mathrm{C}\right)$, degassed $\left(\mathrm{CO}_{2}\right)$ modified McDougall's buffer. Preliminary investigations in our laboratory confirmed that this procedure ensures that small particles are retained in the rumen inoculum. Buffer was prepared on each day of incubation by weighing $9.3 \mathrm{~g}$ of $\mathrm{NaHCO}_{3}$ and $9.8 \mathrm{~g}$ of $\mathrm{NaHPO}_{4}$ into a volumetric flask, followed by the addition of $20 \mathrm{~mL}$ of chloride solution (prepared by diluting $0.47 \mathrm{~g}$ of $\mathrm{NaCl}, 0.57 \mathrm{~g}$ of $\mathrm{KCl}, 0.12 \mathrm{~g}$ of
$\mathrm{MgCl}_{2}$, and $0.04 \mathrm{~g}$ of $\mathrm{CaCl}_{2}$ in deionized water to a final volume of $20 \mathrm{~mL}$ ) and deionized water to a final volume of $1.5 \mathrm{~L}$. Buffered strained ruminal fluid was degassed with $\mathrm{CO}_{2}$ for $10 \mathrm{~min}$.

Fifty milliliters of buffered ruminal fluid; $400 \mathrm{mg}$ of finely ground dry hay; $2.5 \mathrm{~mL}$ of LeA stock emulsions providing 1, 2.5, 5.0, or $10.0 \mathrm{mg}$ of LeA; and $5 \mathrm{mg}$ of nonadecanoic acid (Larodan Fine Chemicals AB) as an internal standard $(19: 0,5 \mathrm{mg} / \mathrm{mL}$ in ethanol) were transferred into $100-\mathrm{mL}$ glass flasks. Ground hay was included as a fermentation substrate, because anaerobes do not generate ATP from LeA (Fievez et al., 2007) and also to provide a site for attachment, as the majority of biohydrogenation occurs on the surface of feed particles (Harfoot et al., 1973a). Flasks, containing rumen fluid, buffer, ground hay, and internal standard (19:0) were also prepared and used as blank samples. Each flask was degassed with $\mathrm{CO}_{2}$, covered with a tight-fitting butyl rubber stopper, and incubated in on a benchtop shaker (Infors HT AG, Bottmingen, Switzerland), operated at a rotation speed of $85 \mathrm{rpm}$ and maintained in the dark at $39^{\circ} \mathrm{C}$ for $0,1.5,3.0,4.5,6.0$, and $9.0 \mathrm{~h}$.

At the end of each designated time point, incubations were stopped by placing the flasks into ice-cold water and the $\mathrm{pH}$ of flask contents was measured. The contents of each flask were frozen $\left(-20^{\circ} \mathrm{C}\right)$, freeze-dried $(\mathrm{B}$. Brown Christ Gamma 2-20; B. Braun Melsungen AG, Melsungen, Germany), weighed, and stored at $-20^{\circ} \mathrm{C}$ until submitted for fatty acid determinations. Incubations were repeated over 5 separate days with 1 flask for each incubation time per treatment.

\section{Fatty Acid Analysis}

Deionized water $(0.5 \mathrm{~mL})$ was added to the $100 \mathrm{mg}$ of freeze-dried flask contents. After adjusting the $\mathrm{pH}$ to 2.0 with $2 M$ hydrochloric acid, lipid was extracted using $4 \mathrm{~mL}$ of a mixture $(3: 2 ; \mathrm{vol} / \mathrm{vol})$ of hexane and isopropanol (Shingfield et al., 2003). Lipid extraction was repeated using the same solvent mixture and both organic phases recovered were combined, washed with deionized water, dried over approximately $200 \mathrm{mg}$ of sodium sulfate, and evaporated to dryness at $45^{\circ} \mathrm{C}$ under a constant stream of nitrogen. Thereafter, NEFA were separated from other lipid fractions by solid-phase extraction using aminopropyl-bonded silica cartridges (1 g, Mega Bond Elut, Varian sample preparation products; Varian Inc., Harbor City, CA). Cartridges were preconditioned at atmospheric pressure with hexane and samples were allowed to penetrate the solid phase before the elution of neutral lipids with hexane and isopropanol (3:1; vol/vol). Nonesterified fatty acids were liberated using a mixture of acetic acid and di- 
ethyl ether $(2: 98 \mathrm{vol} / \mathrm{vol})$ and the column effluent was evaporated to dryness under nitrogen at $45^{\circ} \mathrm{C}$. Methyl esters were prepared by incubation of NEFA with methanolic sulfuric acid $(1 \% \mathrm{vol} / \mathrm{vol})$ at $50^{\circ} \mathrm{C}$ for 30 min (Shingfield et al., 2003). The reaction mixture was washed with aqueous sodium chloride $(5 \% \mathrm{wt} / \mathrm{vol})$ to promote the recovery of FAME in hexane. After washing with aqueous sodium hydrogen carbonate $(2 \% \mathrm{wt} /$ vol) the hexane-soluble phase was dried with sodium sulfate and transferred to glass GC vials.

Fatty acid methyl esters were separated and quantitated using a gas chromatograph (5890, Series II; Hewlett-Packard, Wilmington, DE) equipped with a flame-ionization detector, automatic injector, and a $100-\mathrm{m}$ fused silica capillary column (i.d. $0.25 \mathrm{~mm}$ ) coated with a $0.2-\mu \mathrm{m}$ film of cyanopropyl polysiloxane (CP-SIL 88, Chrompack 7489; Chrompack International BV, Middelburg, the Netherlands). The total FAME profile in a $1-\mu \mathrm{L}$ sample volume at a split ratio of 1:70 was determined using a temperature gradient program with helium as the carrier gas operated at constant pressure $(195 \mathrm{kPa})$ at a flow rate of $0.6 \mathrm{~mL} / \mathrm{min}$. Following sample injection, the column temperature was maintained at $70^{\circ} \mathrm{C}$ for $1 \mathrm{~min}$, increased at a rate of $30^{\circ} \mathrm{C} / \mathrm{min}$ to $170^{\circ} \mathrm{C}$, held for $54 \mathrm{~min}$ and raised to $220^{\circ} \mathrm{C}$ at a rate of $30^{\circ} \mathrm{C} / \mathrm{min}$ and maintained at $220^{\circ} \mathrm{C}$ for 15 min. The total run time was $75 \mathrm{~min}$. Injector and detector temperatures were maintained at 260 and $240^{\circ} \mathrm{C}$, respectively.

Peaks were identified based on retention time comparisons with authentic FAME standards (GLC \#67 and GLC\#74; Nu-Check-Prep Inc., Elysian, MN). Methyl esters not contained in commercially available standards were formally identified based on GC-MS analysis of DMOX derivatives prepared from FAME fractionated according to the degree of unsaturation and configuration of the double bonds by $\mathrm{Ag}^{+}-\mathrm{TLC}$. The TLC plates (Silica gel G, $200 \times 200 \mathrm{~mm}$ and 0.50 mm thickness; No. 1.13894; Merck Co., Darmstadt, Germany) were impregnated with $\mathrm{Ag}^{+}$by immersion in a silver nitrate solution (5\%, wt/vol in acetonitrile) for $20 \mathrm{~min}$. Following the application of approximately $15 \mathrm{mg}$ of lipid, plates were developed with a mixture of hexane and diethyl ether (80:20; vol/vol) and visualized under UV light at $302 \mathrm{~nm}$ (UV trans-illuminator, 2011 Macrovue; LKB, Bromma, Sweden) after spraying with a $0.2 \%$ (wt/vol) solution of $2^{\prime}, 7^{\prime}$-dichlorofluorescein in methanol (Cruz-Hernandez et al., 2004). Five separated bands were individually scrapped off the plates and transferred to clean test tubes. A mixture of geometric isomers of 9,12 18:2 (L-8404; Sigma, St. Louis, MO) of known composition was used to assign the geometry of double bonds for fatty acids recovered in each fraction. Methyl esters were recovered from the silica after the addition of $4 \mathrm{~mL}$ of methanol, $8 \mathrm{~mL}$ of hexane, and 4 $\mathrm{mL}$ of a $6 \%(\mathrm{wt} / \mathrm{vol})$ solution of aqueous sodium chloride (Kawashima and Ohnishi, 2004). After drying over anhydrous sodium sulfate, the hexane soluble phase was evaporated to dryness under nitrogen at room temperature and used to prepare DMOX derivatives.

The DMOX derivatives were prepared from FAME by incubation overnight $(18 \mathrm{~h}$ ) with $250 \mathrm{mg}$ of 2-amino,2methyl-1-propanol under a nitrogen atmosphere according to Fay and Richli (1991), with the exception that a temperature of $170^{\circ} \mathrm{C}$ was used. Once cooled, the reaction mixture was dissolved with $5 \mathrm{~mL}$ of a mixture of diethyl ether and hexane $(1: 1, \mathrm{vol} / \mathrm{vol})$ and washed with $5 \mathrm{~mL}$ of deionized water. Organic extracts were combined, washed with $3 \mathrm{~mL}$ of deionized water, dried over anhydrous sodium sulfate, and evaporated to dryness under nitrogen at room temperature. The DMOX derivatives were dissolved in hexane and analyzed using a gas chromatograph (6890; Hewlett-Packard) equipped with a 100-m CP-Sil 88 fused silica capillary column (Chrompack International BV) and selective quadrupole mass detector (model 5973N; Agilent Technologies Inc., Wilmington, DE) connected to a dedicated computer with ChemStation software installed. Total DMOX profiles in a $4-\mu \mathrm{L}$ sample volume were determined using helium as the carrier gas and the same temperature gradient applied during GC analysis of FAME. The mass spectrometer was operated at $230^{\circ} \mathrm{C}$ in the electron impact ionization mode and mass spectra were recorded under the ionization energy of $70 \mathrm{eV}$. Both the ion source and interface temperatures were maintained at $230^{\circ} \mathrm{C}$.

Interpretation of mass spectra was undertaken according to published guidelines (Spitzer, 1996; Christie, 1998), with an interval of 12 amu between the most intense peaks of clusters of ions containing $n$ and $n-$ 1 carbon atoms being interpreted as cleavage of the double bond between carbon $\mathrm{n}$ and $\mathrm{n}+1$ in the fatty acid moiety. In addition, odd-numbered fragments at $m / z 139,153$, and 167 accompanied by even mass ions at $m / z 138,152$, and 166 in the mass spectrum of DMOX derivatives were used as diagnostic ions to locate double bonds at $\Delta 4, \Delta 5$, and $\Delta 6$, respectively (Spitzer, 1996; Christie, 1998). When available, the deduced fatty acid structure was verified by comparison with an online reference spectra library (http:// lipidlibrary.aocs.org/ms/masspec.html). Double-bond geometry was deduced based on the relative abundance of FAME in each band recovered by $\mathrm{Ag}^{+}-\mathrm{TLC}$, retention time during GC analysis, and the elution order of geometric isomers of 9,12 18:2 methyl esters.

The distribution of CLA isomers formed during in vitro incubations was determined using an HPLC system (Model 1090; Hewlett-Packard) equipped with 4 silver 
impregnated silica columns (ChromSpher 5 Lipids, 250 $\times 4.6 \mathrm{~mm} ; 5-\mu \mathrm{m}$ particle size; Varian Ltd., Walton-onThames, UK) coupled in series. Methyl esters of CLA were separated under isocratic conditions at $22^{\circ} \mathrm{C}$ using $0.1 \%$ ( $\mathrm{vol} / \mathrm{vol}$ ) acetonitrile in heptane at a flow rate of $1 \mathrm{~mL} / \mathrm{min}$ and monitoring column effluent at $233 \mathrm{~nm}$ (Shingfield et al., 2003). Typical injection volumes were 10 to $20 \mu \mathrm{L}$, representing $<250 \mu \mathrm{g}$ of lipid. Identification of CLA isomers was performed using commercially available CLA methyl ester standards (Matreya Inc., Pleasant Gap, PA; Sigma) and was verified by crossreferencing with the elution order reported in the literature (Delmonte et al., 2005) using cis-9,trans-11 CLA as a landmark isomer. Concentrations of specific conjugated isomers were calculated based on proportionate peak area responses determined by HPLC and the sum of trans-7,cis-9 CLA; trans-8,cis-10 CLA; and cis-9,trans-11 CLA determined as a single peak during $\mathrm{GC}$ analysis.

\section{Calculations and Statistical Analysis}

Amounts of fatty acids expressed as milligrams per flask were determined based on measurements of fatty acid composition and the fatty acid content of incubation residues determined using nonadecanoic acid as an internal standard. Concentrations of fatty acids were corrected by subtracting the amount of fatty acids measured in corresponding blank samples containing rumen fluid and all reagents except LeA from the amounts of fatty acids determined for all treatment samples. Corrected values were used to calculate LeA disappearance and the rates of biohydrogenation intermediate and end-product formation. Kinetic parameters of LeA disappearance expressed as a percentage of the initial amount added were estimated according to a first-order exponential model (Ørskov and McDonald, 1979), defined as

$$
\mathrm{Q}_{\mathrm{t}}=\mathrm{a}+\mathrm{b}\left(1-\exp ^{-\mathrm{ct}}\right)
$$

where $Q_{t}$ represents the percentage of LeA that disappears in time $t(h)$, expressed as a percentage of the initial amount; $a$ is the disappearance of LeA at time 0 $\mathrm{h}$ (expressed as a percentage of the initial amount); $\mathrm{b}$ is the percentage of LeA that can potentially disappear during incubations with ruminal fluid (expressed as the percentage of the initial amount); and $\mathrm{c}$ is the fractional rate of fraction $\mathrm{b}$ (expressed as $1 / \mathrm{h}$ ). Parameters $\mathrm{a}, \mathrm{b}$, and $\mathrm{c}$ for LeA inclusion levels of 1.0, 2.5, and 5.0 $\mathrm{mg} /$ flask and for each day of incubation were computed using the NLIN procedure of the SAS (version 9.2; SAS Institute Inc., Cary, NC). Disappearance of LeA over time at the highest level of inclusion (10 $\mathrm{mg} /$ flask) was found to be linear and, therefore, the parameters to describe the kinetics of LeA biohydrogenation were evaluated by linear regression.

Measurements of the amount of biohydrogenation intermediates and 18:0 formed over time during incubations of incremental amounts of LeA with rumen fluid for each day of incubation were averaged to generate mean values for each incubation time per treatment for each day of incubation. These data were used to estimate fractional rates of transfer among fatty acid pools using WinSAAM software (Version 3.0.7; New Bolton Center, Biostatistics Unit, University of Pennsylvania, Philadelphia; available at http://www.winsaam.com) according to the model outlined by Ribeiro et al. (2007). Representation of fatty acid pools used to estimate fractional rates of LeA biohydrogenation is shown in Figure 1. Intermediates formed during the initial biohydrogenation of LeA were assigned to 1 of 2 pools; CLA and nonconjugated (NC) 18:2, both of which were assumed to be reduced to 18:1 intermediates. Isomers of CLA are known to be reduced to 18:1 isomers during incubations with ruminal bacteria (McKain et al., 2010) and trans 18:1 can be converted to cis 18:1 and vice versa during incubations with ruminal fluid (Mosley et al., 2002; Proell et al., 2002). Therefore, all 18:1 products were considered as a single fatty acid pool (Figure 1).

Data on the incubation $\mathrm{pH}$, rates of LeA disappearance, fractional rates, and amounts of intermediates that accumulated over time during incubations of LeA with strained ruminal fluid were analyzed by ANOVA for repeated measures using the mixed linear model procedure of SAS with a statistical model that included the effects of LeA addition, incubation time, and their interaction, with incubation day as the repeated variable assuming a compound symmetry covariance structure fitted on the basis of Akaike information and Schwarz Bayesian model-fit criteria. Sums of squares for treatment effects were further separated using polynomial contrasts into single degree-of-freedom comparisons to test for the significance of linear, quadratic, and cubic components of the effects of LeA addition on disappearance rates, accumulation of intermediates, and appearance of 18:0 end product. Least squares means are reported and treatment effects declared significant at $P<0.05$.

\section{RESULTS}

\section{$p H$}

During the course of incubations, the $\mathrm{pH}$ of fermentation flask contents decreased from 6.6 to 6.2. The $\mathrm{pH}$ of incubation flasks was not altered by the initial amount 


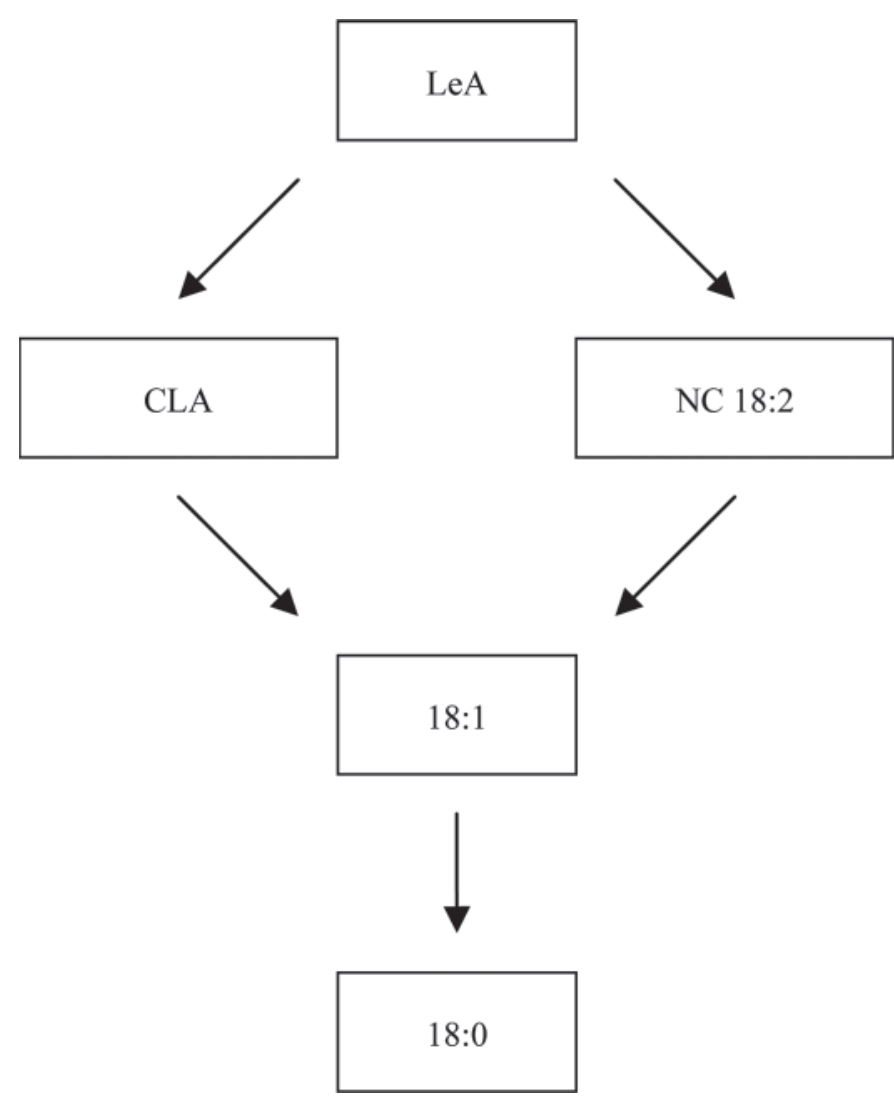

Figure 1. Model of biohydrogenation of linoleic acid (LeA) during incubations with strained ruminal fluid. Boxes represent fatty acid pools and arrows indicate the transfer of fatty acids between pools during biohydrogenation. Linoleic acid, geometric isomers of conjugated linoleic acid (CLA), nonconjugated 18:2 isomers (NC 18:2), cis and trans 18:1 intermediates (18:1), and stearic acid (18:0).

of LeA $(P>0.05)$ or due to interactions between the amount of added LeA and incubation time $(P>0.05$; Figure 2).

\section{Disappearance of LeA, Accumulation of Biohydrogenation Intermediates, and Formation of Stearic Acid}

Incubations with strained ruminal fluid resulted in extensive biohydrogenation of LeA. At the end of $9 \mathrm{~h}$ incubations, 98.1, 97.6, 98.0, and 89.8\%, respectively, of $1.0,2.5,5.0$, and $10.0 \mathrm{mg}$ of added LeA disappeared. A significant amount of LeA was found to disappear several minutes after the start of incubations with strained ruminal fluid, but the proportion of LeA disappearing at time zero decreased linearly $(P<0.05)$ with LeA addition (Table 1). Furthermore, increases in LeA addition enhanced linearly $(P=0.001)$ the percentage of potential LeA disappearance from 71.2 to $87.4 \%$ but decreased the fractional rate of this fraction from 54.1 to $37.5 \% / \mathrm{h}$ (Table 1). At the highest level of substrate

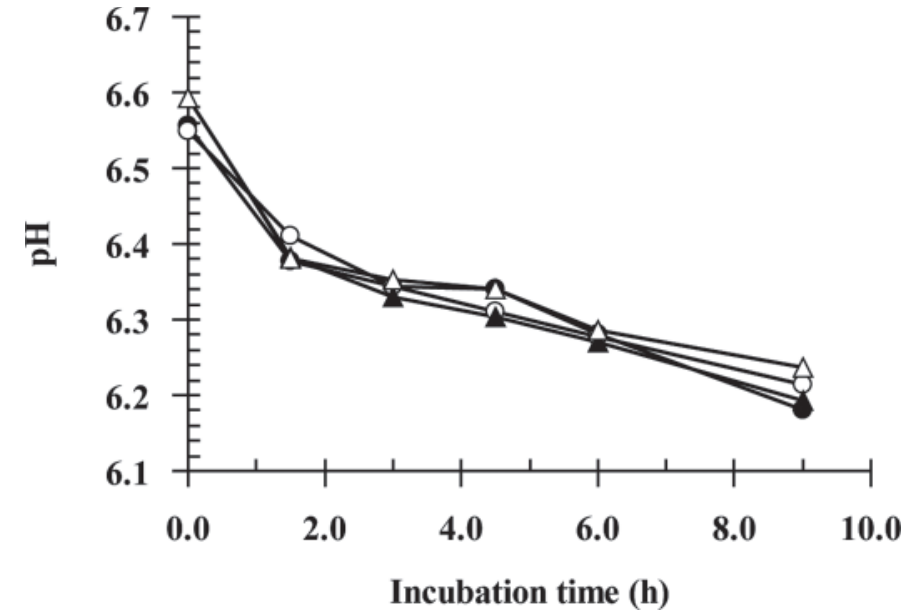

Figure 2. Temporal changes in mean $\mathrm{pH}$ of flask contents during incubations of $1.0(\bullet), 2.5(\bigcirc), 5.0(\mathbf{\Delta})$, and $10.0(\Delta) \mathrm{mg}$ of linoleic acid with strained ruminal fluid. Values represent least squares means $(\mathrm{n}=5 ; \mathrm{SEM}=0.018)$.

addition, the rate of LeA disappearance was found to be constant at $7.6 \% / \mathrm{h}$ over the 9 -h incubation period (Figure 3). The sum of LeA, 18-carbon unsaturated biohydrogenation intermediates, and 18:0 averaged $1.15,2.50,4.97$, and $10.08 \mathrm{mg} /$ flask over the course of 0 to 9 -h incubations with $1.0,2.5,5.0$, and $10.0 \mathrm{mg}$ of added LA, respectively. Incubations of incremental amounts of LeA resulted in a dose- and time-dependent accumulation of 18:2 isomers, 18:1 intermediates, and 18:0 (Table 2; Figure 4). Production of 18:0 increased in response to LeA addition, but at the highest level, complete biohydrogenation of LeA to 18:0 decreased

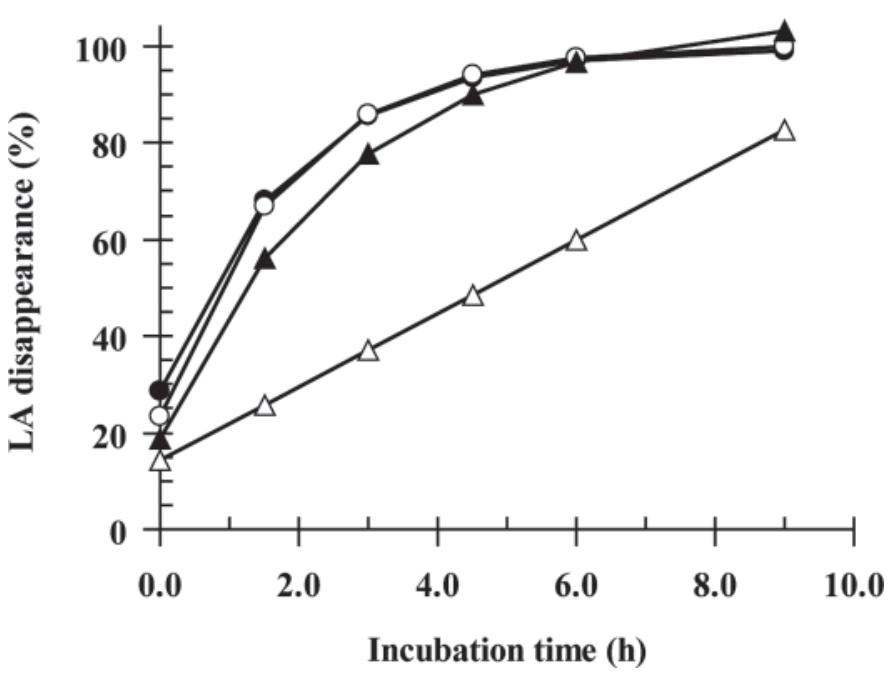

Figure 3. Temporal changes in the disappearance of linoleic acid $(\mathrm{LA})$ during incubations of $1.0(\bullet), 2.5(\bigcirc), 5.0(\mathbf{\Delta})$, and $10.0(\Delta) \mathrm{mg}$ of added linoleic acid with strained ruminal fluid. Values represent least squares means $(\mathrm{SEM}=4.97 \%)$. 
Table 1. Adjusted parameters of a single available pool, first-order kinetic model describing the disappearance of linoleic acid (LeA) during incubations with strained ruminal fluid

\begin{tabular}{|c|c|c|c|c|c|c|}
\hline \multirow[b]{2}{*}{ Parameter $^{1}$} & \multicolumn{3}{|c|}{ LeA addition $(\mathrm{mg} / \text { flask })^{2}$} & \multirow[b]{2}{*}{ SEM } & \multicolumn{2}{|c|}{$P$-value ${ }^{3}$} \\
\hline & 1.0 & 2.5 & 5.0 & & $\mathrm{~L}$ & Q \\
\hline a & 28.5 & 23.4 & 18.6 & 2.89 & 0.044 & 0.710 \\
\hline $\mathrm{b}$ & 71.2 & 77.0 & 87.4 & 2.07 & 0.001 & 0.908 \\
\hline $\mathrm{c}$ & 0.541 & 0.553 & 0.375 & 0.0349 & 0.007 & 0.124 \\
\hline$a+b$ & 99.7 & 100.4 & 106 & 1.27 & 0.006 & 0.315 \\
\hline
\end{tabular}

${ }^{1}$ Estimated using a first-order exponential model $Q_{t}=a+b\left(1-e^{-c t}\right)$, where $Q_{t}$ is the percentage of LeA disappearance at time $\mathrm{t}(\mathrm{h})$, a is the disappearance of LeA at time $0 \mathrm{~h}$ (\% of the initial amount), $\mathrm{b}$ is the percentage of LeA that can potentially disappear during incubations with strained ruminal fluid (\% of the initial amount), $\mathrm{c}$ is the fractional rate of fraction $\mathrm{b}$ (expressed in $1 / \mathrm{h}$ ), and $\mathrm{a}+\mathrm{b}$ represents the maximum percentage of LeA disappearance (\% of the initial amount).

${ }^{2}$ Disappearance during incubations with $10 \mathrm{mg}$ of LeA with strained ruminal fluid was linear according the relationship $\mathrm{Q}_{\mathrm{t}}=14.2 \pm 0.626(\%)+7.6 \pm 0.0102(\% / \mathrm{h}) \times \mathrm{t}(\mathrm{h})\left(\mathrm{n}=6, \mathrm{r}^{2}=0.957, P<0.001\right)$, where $\mathrm{Q}_{\mathrm{t}}$ is the percentage of LeA disappearance at time t.

${ }^{3}$ Significance of linear (L) and quadratic (Q) components of the response to LeA addition on first-order kinetic model parameters. Cubic responses to the amount of LeA incubated on model parameters were not significant $(P>0.05)$.

(Figure 4). Percentages of the initial amount of LeA incubated recovered as 18:0 after 9-h incubations were $73.6,65.0,57.3$, and $10.7 \%$ for $1.0,2.5,5.0$, and $10.0 \mathrm{mg}$ of added LeA, respectively. Decreases in the complete hydrogenation of LeA were associated with linear or quadratic increases $(P<0.001)$ in the accumulation of CLA, NC 18:2, and 18:1 intermediates (Table 2). Increasing the amounts of LeA incubated with strained ruminal fluid resulted in a linear $(P<0.001)$ increase in the ratio of total NC 18:2:total CLA intermediates (mean 4.55, 6.58, 10.9, and 22.0, SEM = 3.94, for 1.0, 2.5, 5.0, and $10.0 \mathrm{mg}$ of added LeA, respectively).

\section{Formation of Octadecadienoic Intermediates}

Incremental addition of LeA resulted in a linear $(P$ $<0.01)$ increase in the accumulation of cis-9,trans-11 CLA and trans-10,cis-12 CLA (Table 3) during incubations with strained ruminal fluid. In addition to these 2 main conjugated intermediates, incubations of LeA also increased linearly $(P<0.05)$ the accumulation of cis-9,cis-11 CLA; cis-10,cis-12 CLA; trans-9,trans-11 CLA; and trans-10,trans-12 CLA (Table 3). Temporal changes in the accumulation of $\Delta 9,11$ and $\Delta 10,12$ geometric isomers of CLA over 9-h incubations of incremental amounts of LeA with strained ruminal fluid are shown in Figure 5. Formation of cis-9,trans-11 CLA was instantaneous following the addition of LeA to the fermentation flask, and was rapidly hydrogenated, whereas trans-10,cis-12 CLA tended to accumulate with the highest abundance observed after $1.5 \mathrm{~h}$ (Figure 5). At the highest level of LeA addition, cis-9,trans-11 CLA accumulated after $3 \mathrm{~h}$ (Figure 5).

Analysis of FAME, prepared from NEFA extracted from fermentation flasks, revealed the appearance of several unknown peaks eluting between 19:0 and LeA in the GC chromatogram (Figure 6). Initial identification of unusual fatty acids eluting in this region was based on retention time comparisons with authentic standards, but because of the limited number of ref-

Table 2. Effect of incremental addition of linoleic acid (LeA) on mean accumulation of biohydrogenation intermediates and end products during incubations with strained ruminal fluid

\begin{tabular}{|c|c|c|c|c|c|c|c|}
\hline \multirow{2}{*}{$\begin{array}{l}\text { Amount } \\
(\mu \mathrm{g} / \text { flask })^{1}\end{array}$} & \multicolumn{4}{|c|}{ LeA addition (mg/flask) } & \multirow[b]{2}{*}{ SEM } & \multicolumn{2}{|c|}{$P$-value ${ }^{2}$} \\
\hline & 1.0 & 2.5 & 5.0 & 10.0 & & $\mathrm{~L}$ & Q \\
\hline LeA & 252 & 624 & 1,444 & 4.964 & 204.7 & $<0.001$ & $<0.001$ \\
\hline$\Sigma$ CLA & 138 & 164 & 184 & 305 & 24.3 & $<0.001$ & 0.441 \\
\hline$\Sigma 18: 2^{3}$ & 78.9 & 128 & 322 & 963 & 51.5 & $<0.001$ & 0.020 \\
\hline $18: 1$ & 291 & 795 & 1,855 & 3,517 & 149.8 & $<0.001$ & 0.505 \\
\hline $18: 0$ & 402 & 804 & 1,193 & 344 & 187.6 & 0.289 & $<0.001$ \\
\hline
\end{tabular}

${ }^{1}$ Values represent least squares means measured as a difference relative to the control determined after 0-, 1.5-, 3.0-, 4.5-, 6.0-, and 9.0-h incubations $(\mathrm{n}=5)$. CLA = conjugated linoleic acid.

${ }^{2}$ Significance of linear (L) and quadratic (Q) components of the response to LeA addition. Cubic responses to the amount of LeA incubated were not significant $(P>0.05)$.

${ }^{3}$ Refers to 18:2 intermediates, excluding isomers of CLA and LeA. 

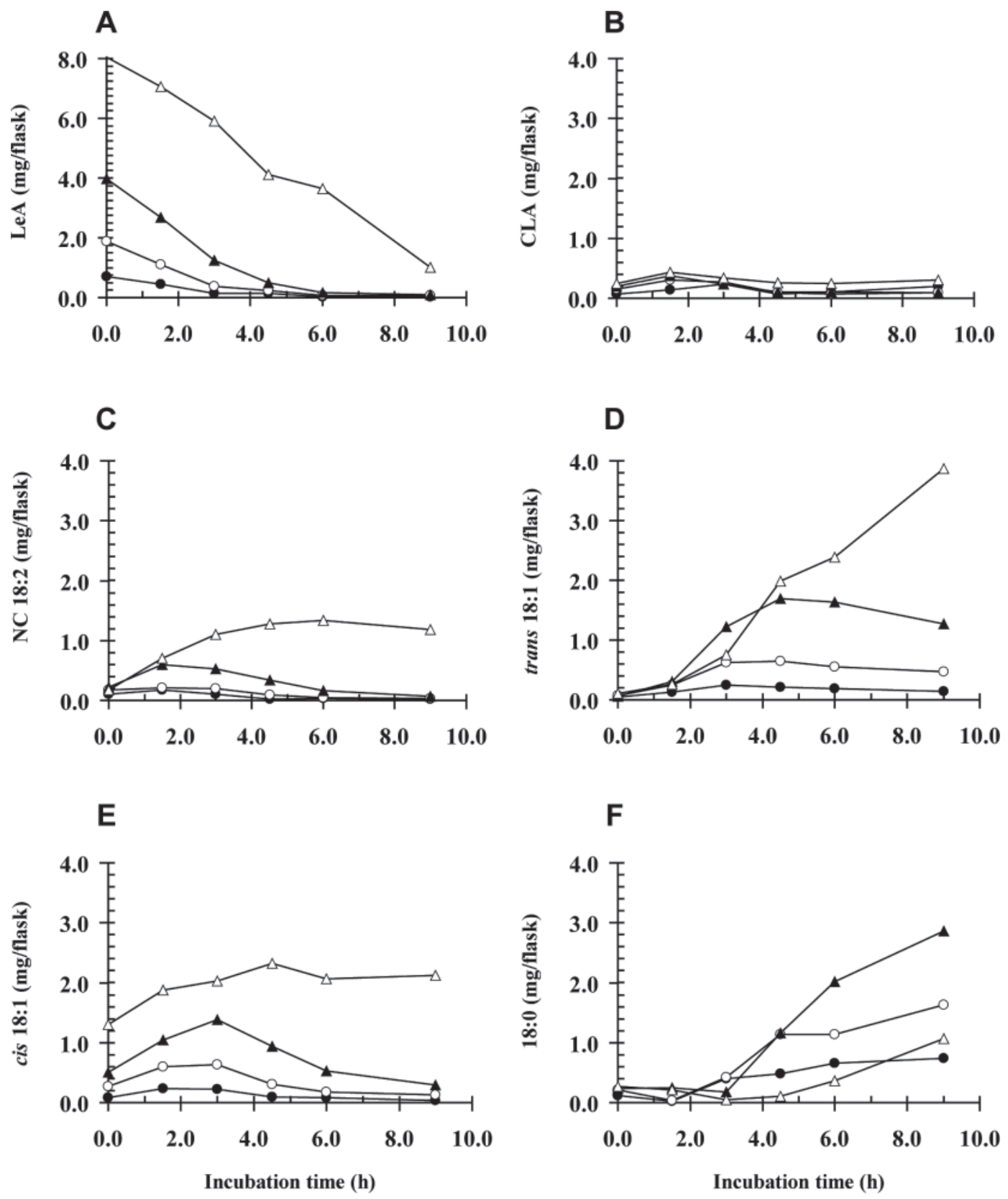

Figure 4. Temporal changes in the accumulation of (A) linoleic acid (LeA); (B) conjugated linoleic acid (CLA); (C) nonconjugated 18:2 (NC 18:2); (D) trans 18:1; (E) cis 18:1; and (F) stearic acid (18:0) during incubations of $1.0(\bullet), 2.5(O), 5.0(\mathbf{\Delta})$, and $10.0(\Delta)$ mg of LeA with strained ruminal fluid. Values represent least squares means $(\mathrm{n}=5$; SEM $=0.34,0.30,0.06,0.27,0.17$, and 0.29 mg/flask for LeA, CLA, NC 18:2, trans 18:1, cis 18:1, and 18:0, respectively).

erence materials, few peaks could be identified with a high degree of confidence. The GC-MS analysis of novel intermediates as methyl esters revealed that all exhibited a molecular ion at $m / z 294$, confirming an 18-carbon structure with 2 double bonds. Fractionation of FAME by $\mathrm{Ag}^{+}$-TLC enabled 6 distinct bands to be separated, corresponding to methyl esters of saturated fatty acids, trans-monoenoic acids, and cis-monoenoic acids, and mixtures of trans,trans; trans,cis; cis,trans; and cis, cis dienoic acids. Partial GC chromatograms indicating the separation of 18:2 isomers recovered after $\mathrm{Ag}^{+}$-TLC fractionation are shown in Figure 7.

The structure of unusual 18:2 isomers were formally identified based on GC-MS analysis of DMOX derivatives prepared from each FAME fraction recovered after $\mathrm{Ag}^{+}$-TLC. Characteristic ion fragments in the mass 
Table 3. Effect of incremental addition of linoleic acid (LeA) on mean accumulation of isomers of conjugated linoleic acid (CLA) during incubations with strained ruminal fluid

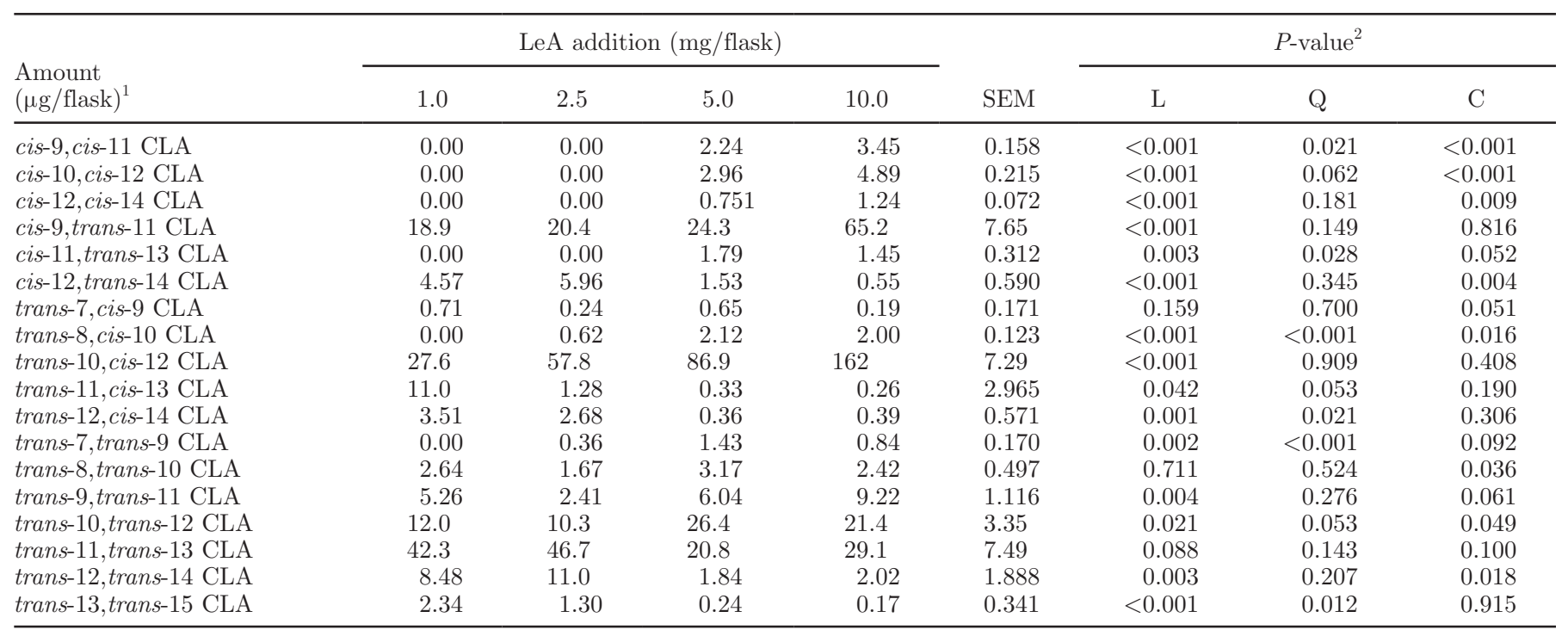

${ }^{1}$ Values represent least squares means measured as a difference relative to the control determined after 0-, 1.5-, 3.0-, 4.5-, 6.0-, and 9.0-h incubations $(\mathrm{n}=5)$.

${ }^{2}$ Significance of linear (L), quadratic (Q), and cubic (C) components of the response to LeA addition.

spectrum of DMOX derivatives used to identify the structure of unusual 18:2 isomers are listed in Table 4. Spectra of all DMOX derivatives of 18:2 isomers contained abundant ions at $m / z 113$ and 126 and a molecular ion at $m / z 333$ (Table 4). A prominent fragment at $m / z$ at 113 arises from a McLafferty rearrangement ion formed by the migration of the $\gamma$-hydrogen, followed by cleavage between carbon atoms 2 and 3, whereas the abundant ion at $m / z$ at 126 is thought to be formed due to a cyclization-displacement reaction and cleavage between carbon atoms 4 and 5 (Spitzer, 1996; Christie, 1998). The mass spectrum of the DMOX derivative of cis-7,cis-12 18:2 contained 2 pairs of ion fragments separated by 12 -amu gaps at $m / z 168$ and 180 , and 236 and 248 , and 14-amu intervals between ions at $m / z 194$, 208, and 222, and 262, 276, 290, 304, and 318 locating double bonds at $\Delta 7$ and $\Delta 12$ (Figure $8 \mathrm{~A}$ ). Double-bond positions for isomers of $\Delta 8,12$ 18:2 were indicated based on 12-amu intervals between $\mathrm{m} / z 182$ and 194, and 236 and 248 (Figure 8B), and a prominent ion at $\mathrm{m} / z 222$ that represents cleavage between carbon atoms 9 and 10 (i.e., at the center of the dimethylene-interrupted ethylenic bond system).

Because GC-MS analysis of DMOX derivatives does not discriminate between geometric isomers, the double bond geometry of most NC 18:2 intermediates could be confirmed based on the appearance of FAME in bands separated by $\mathrm{Ag}^{+}$-TLC. However, the methyl ester of $\Delta 4,12$ 18:2 was not recovered in either cis, cis 18:2 fraction (Figure 7) suggesting this isomer contained at least one trans double bond, whereas the retention time and relative elution order of this isomer during GC analysis of FAME (Figure 6) would tend to implicate a cis, cis double bond configuration.

Complementary $\mathrm{Ag}^{+}$-TLC and GC-MS analysis of DMOX derivatives confirmed that the biohydrogenation of LeA by strained ruminal fluid under the specified conditions of this experiment resulted in the formation of $\Delta 4,12$ 18:2; cis-5,cis-12 18:2; cis-6, cis-12 18:2; cis-7,cis-12 18:2; cis-8,cis-12 18:2; cis-9,trans-12 18:2; trans-8,cis-12 18:2; and trans-9,cis-12 18:2. Addition of incremental amounts of LeA resulted in linear or quadratic $(P<0.01)$ increases in the accumulation of cis-5,cis-12 18:2 + cis-6,cis-12 18:2; cis-7,cis-12 18:2; cis-8,cis-12 18:2; trans-8,cis-12 18:2+ $\Delta 4,12$ 18:2; and trans-9,cis-12 18:2 during incubations with strained ruminal fluid (Table 5). Increases in the amount of these biohydrogenation intermediates during incubations with $1.0,2.5$, and $5.0 \mathrm{mg}$ of LeA reached a plateau after 1.5 or $3 \mathrm{~h}$, but decreased thereafter (Figure 9). A mixture of trans-8,cis-12 18:2 and $\Delta 4,12$ was found to accumulate immediately after LeA was added to strained ruminal fluid. Incubations with $10 \mathrm{mg}$ of LeA resulted in the accumulation of all identified NC 18:2 isomers over time (Figure 9).

\section{Formation of Octadecenoic Intermediates}

Isomers of 18:1 represented the most abundant intermediates to accumulate during incubations of LeA 

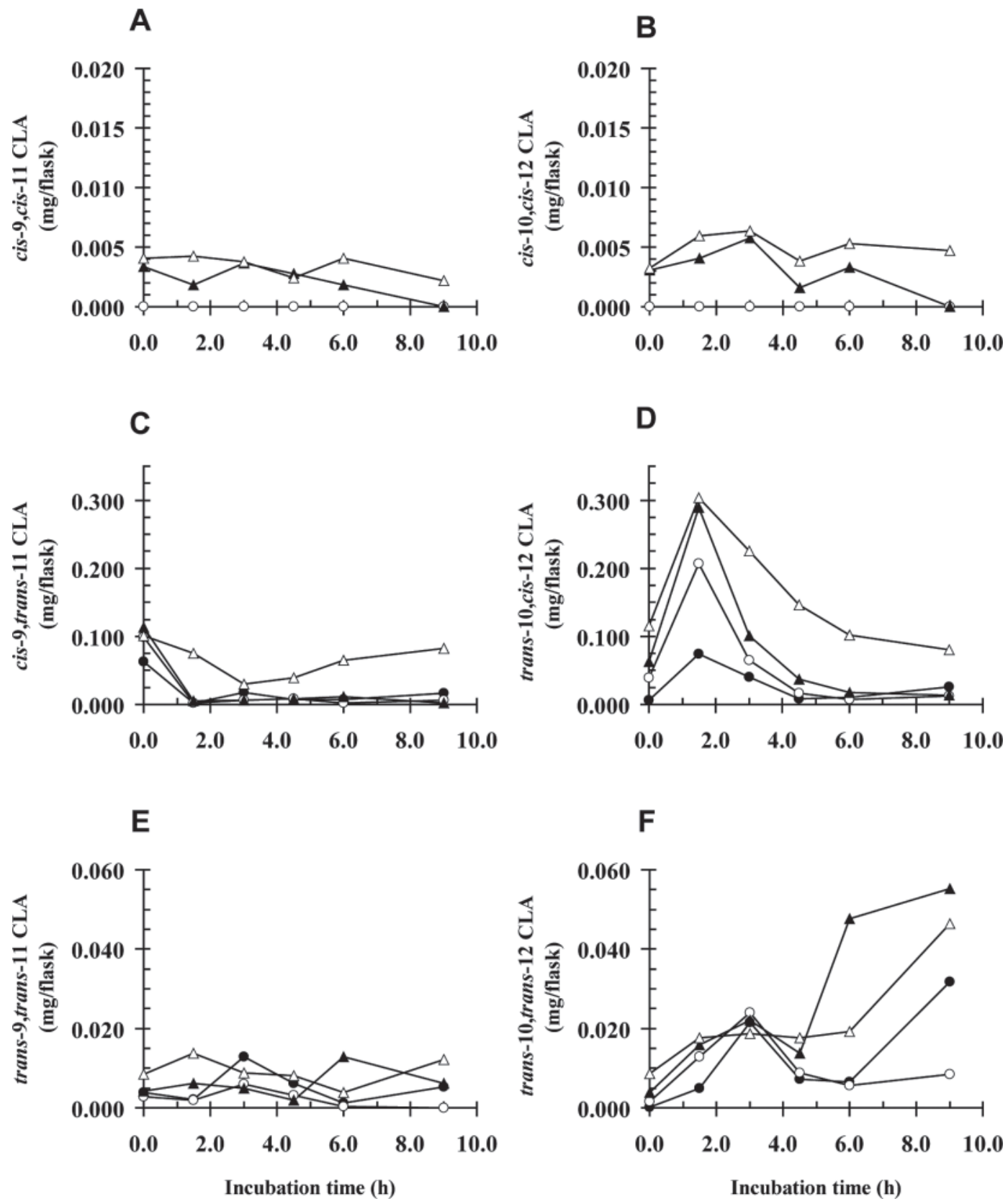

Figure 5. Temporal changes in the accumulation of (A) cis-9,cis-11 conjugated linoleic acid (CLA); (B) cis-10, cis-12 CLA; (C) cis-9,trans-11 CLA; (D) trans-10,cis-12 CLA; (E) trans-9,trans-11 CLA; and (F) trans-10,trans-12 CLA during incubations of $1.0(\bullet), 2.5(\mathrm{O}), 5.0(\mathbf{\Delta})$, and $10.0(\Delta) \mathrm{mg}$ of linoleic acid with strained ruminal fluid. Values represent least squares means $(\mathrm{n}=5 ; \mathrm{SEM}=0.00046,0.00062,0.022,0.021$, 0.0032, and 0.0094 mg/flask for cis-9,cis-11 CLA; cis-9,trans-11 CLA; trans-9,trans-11 CLA; cis-10,cis-12 CLA; trans-10,cis-12 CLA; and trans10,trans-12 CLA, respectively).

with strained ruminal fluid (Table 6). Addition of 1.0, 2.5 , and $5.0 \mathrm{mg}$ of LeA resulted in the appearance of cis-9 18:1; cis-12 18:1; trans-10 18:1; trans-11 18:1; and trans-13,14 18:1 as the main 18:1 intermediates, whereas cis-9 18:1, cis-12 18:1, trans-10 18:1, and trans-11 18:1 were the major 18:1 isomers to accumulate during incubations with $10 \mathrm{mg}$ of LeA. Temporal changes in the accumulation of cis-9 18:1, cis-12 18:1, trans-9 18:1, trans-10 18:1, trans-11 18:1, and trans-12 18:1 are shown in Figure 10. Following the addition of LeA to the fermentation flask, cis-9 18:1 was found to be formed instantaneously and subsequently hydrogenated, whereas cis-12 18:1 accumulated after $1.5 \mathrm{~h}$, depending on the amount of added LeA (Figure 10). Addition of $10 \mathrm{mg}$ 


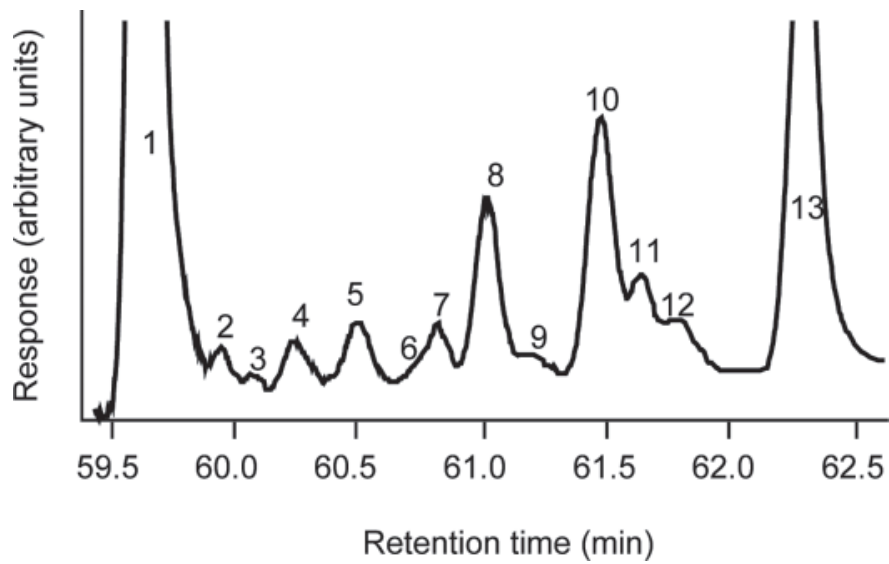

Figure 6. Partial gas chromatogram indicating the separation of 18:2 isomers formed during 4.5-h incubations of $10 \mathrm{mg}$ of linoleic acid with strained ruminal fluid. Isomers were identified based on silver-ion thin-layer chromatography fractionation of fatty acid methyl esters and electron impact ionization spectra obtained by GC-MS analysis of corresponding 4,4-dimethyloxaline derivatives. Peak identification: $1=19: 0 ; 2=$ trans-11,trans-15 18:2; $3=$ trans-9,trans $-1418: 2 ; 4=$ trans-9,trans-12 18:2; $5=$ unresolved trans-8,cis-12 $18: 2$ and $\Delta 4,12$ $18: 2 ; 6=$ cis-5,cis-12 18:2; $7=$ cis-6,cis-12 18:2; $8=$ cis-7,cis-12 18:2; $9=$ cis-9,trans-12 18:2; $10=$ cis-8,cis-12 18:2; $11=$ trans -9, cis-12 18:2; $12=$ trans-11,cis-15 $18: 2 ;$ and $13=$ cis- 9, cis-12 18:2.

of LeA caused cis-12 18:1 to accumulate over the course of the 9-h incubations (Figure 10). Incubation of 1.0, 2.5 , and $5.0 \mathrm{mg}$ of LeA was associated with an initial increase in the amounts of trans-10 18:1 and trans-11 18:1 in fermentation flasks, but the amounts remained relatively constant after $1.5 \mathrm{~h}$. In contrast, addition of $10.0 \mathrm{mg}$ of LeA resulted in a gradual increase in trans-10 18:1 in fermentation flasks up to $4.5 \mathrm{~h}$ and the progressive accumulation of trans-11 18:1 during the course of incubations with strained ruminal fluid. Increasing the amounts of added LeA resulted in a linear $(P=0.021)$ increase in the mean ratio of trans-10 18:1:trans-11 18:1 in fermentation flasks over the 9-h incubation period $(0.787,0.889,1.076$, and 1.671 , SEM $=0.315$, for 1.0, 2.5, 5.0, and $10.0 \mathrm{mg}$ of added LeA, respectively). Incubations of LeA with strained rumen fluid also resulted in a dose-dependent increase $(P<$ $0.01)$ in the accumulation of trans- $6,-7,-8,-9,-12,-13$ and, -14 18:1 (Table 6). Changes in the accumulation of trans-6, -7 , and -8 18:1 and trans-9 18:1 over the course of incubations with LeA followed the same pattern as observed for trans-10 18:1 (data not presented). At lower levels of LeA addition, trans-12 18:1 accumulated in fermentation flasks after $3 \mathrm{~h}$, with the amounts remaining relatively constant thereafter, whereas the addition of $10 \mathrm{mg}$ of LeA resulted in a cumulative increase in trans-12 18:1 over time (Figure 10). Temporal changes in the appearance of trans-13 and -14 18:1 followed the same pattern as trans-12 18:1 (data not presented).
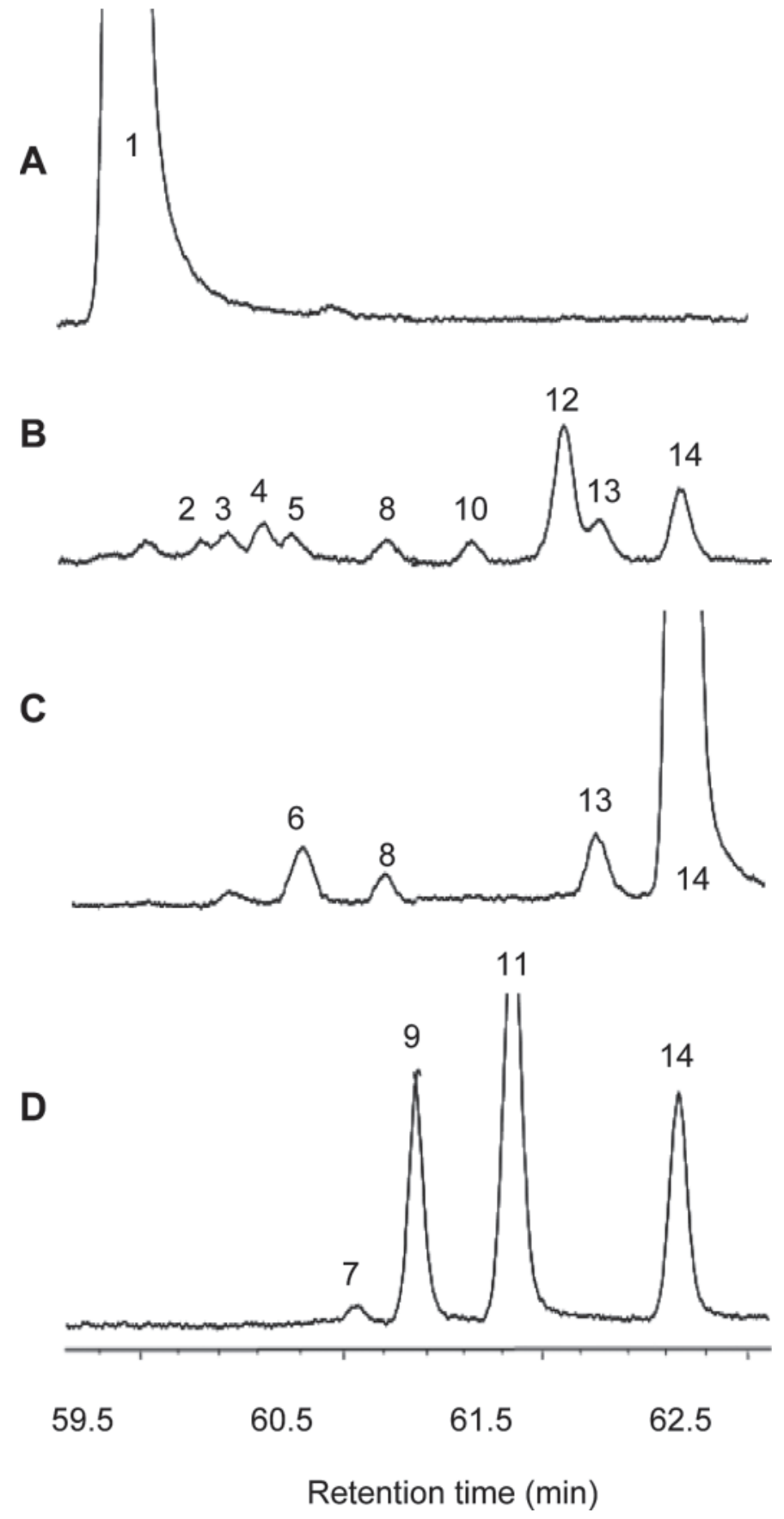

Figure 7. Partial gas chromatogram of the bands recovered after silver-ion thin-layer chromatography separation of fatty acid methyl esters prepared from incubations of linoleic acid-strained ruminal fluid containing saturates (A); cis,trans; trans, cis; and trans,trans 18:2 (B); cis, cis 18:2 (C); and cis,cis 18:2 (D) isomers. Peak identification: 1 $=19: 0 ; 2=$ trans-11,trans $-1518: 2 ; 3=$ trans- 9 ,trans $-1418: 2 ; 4=$ trans-9,trans- $1218: 2 ; 5=4,1218: 2 ; 6=$ trans- 8 ,cis- $1218: 2 ; 7=$ cis5, cis-12 $18: 2 ; 8=$ cis- 6, cis-12 $18: 2 ; 9=$ cis- 7 ,cis-12 $18: 2 ; 10=$ cis9, trans-12 18:2; $11=$ cis-8,cis-12 18:2; $12=$ trans- 9, cis- $1218: 2 ; 13=$ trans-11,cis-15 18:2; and $14=$ cis-9,cis-12 18:2. 
Table 4. Characteristic ion fragments recorded during GC-MS analysis of 4,4-dimethyloxazoline derivatives of unusual octadecadienoic acids formed during incubations of linoleic acid with strained ruminal fluid

\begin{tabular}{ll}
\hline Fatty acid & Characteristic ion fragments $(m / z, \text { relative intensity })^{1}$ \\
\hline$\Delta 4,1218: 2^{2}$ & $113(100), 126(54), 139(6), 166(35), 180(54), 222(46), 236(3), 248(6), 333\left(9, \mathrm{M}+{ }^{3}\right)$ \\
cis-5 cis-12 18:2 $_{\text {cis-6, } \text { cis-12 18:2 }}$ & $113(100), 126(9), 153(18), 166(12), 180(26), 236(4), 248(2), 333(10, \mathrm{M}+)$ \\
cis-7, cis-12 18:2 & $113(59), 126(100), 167(16), 180(20), 194(33), 236(3), 248(3), 333(6, \mathrm{M}+)$ \\
cis-8, cis-12 18:2 & $113(81), 126(100), 166(11), 168(8), 180(90), 194(10), 208(30), 222(54), 236(3), 248(4), 333(5, \mathrm{M}+)$ \\
trans-8, cis-12 18:2 & $113(40), 126(53), 182(7), 194(2), 208(3), 222(100), 236(3), 248(2), 333(7, \mathrm{M}+)$ \\
trans-9, cis-12 18:2 & $113(42), 126(42), 182(6), 194(1), 208(3), 222(100), 236(6), 248(2), 333(9, \mathrm{M}+)$ \\
\hline
\end{tabular}

${ }^{1}$ Mass spectra were recorded at an ionization energy of $70 \mathrm{eV}$ during analysis of 4,4-dimethyloxazoline derivatives on a 100-m CP-Sil 88 fused silica capillary column (Chrompack International BV, Middelburg, the Netherlands) using a temperature gradient program and helium as the carrier gas operated at constant pressure $(142.6 \mathrm{kPa})$ at a flow rate of $0.6 \mathrm{~mL} / \mathrm{min}$.

${ }^{2}$ Double bond geometry indeterminate.

${ }^{3}$ Molecular ion.

\section{Kinetics of LeA Biohydrogenation}

Estimation of transfer rates among fatty acid pools based on the appearance of biohydrogenation intermediates during incubations of 1.0 and $2.5 \mathrm{mg}$ of LeA with strained ruminal fluid using the multiple-pool, first-order kinetic model were not possible due to a small CLA pool size, principally due to the lack of cis-9,trans-11 CLA accumulation in fermentation flasks (Figure 5). Despite attempts to estimate the percentage of added LeA initially isomerized to cis-9, trans-11 CLA transfer rates between pools could not be resolved. Accumulation of intermediates formed during incubations of 5 and $10 \mathrm{mg}$ of LeA with strained ruminal fluid were able to be fitted to the dynamic multi-compartmental model (Figure 1), and indicated that the transfer rate of LeA to the CLA pool (mean 19.1 and $7.3 \% / \mathrm{h}$, respectively) was almost identical to the rate of LeA transfer to the NC 18:2 isomer pool (corresponding values 18.1 and $7.3 \% / \mathrm{h}$ ). However, the rates of transfer from the CLA to $18: 1$ pool $(138$ and $103 \% / \mathrm{h}$, for 5.0 and $10.0 \mathrm{mg}$ of LeA, respectively) were higher compared with the corresponding rates of transfer from the NC 18:2 to 18:1 pool (63.7 and $20.2 \% / \mathrm{h})$. Furthermore, increases in LeA addition had much smaller inhibitory effects on the rate of transfer from the CLA to 18:1 pool compared with the rate of transfer from the NC 18:2 to 18:1 pool. The transfer rates from the 18:1 to 18:0 pool were estimated to be lower than other steps of LeA biohydrogenation by strained ruminal fluid and found to be decreased by increases in LeA addition (14.6 and $1.50 \% / \mathrm{h}$ for incubations with 5.0 and $10.0 \mathrm{mg}$ of LeA, respectively).

\section{DISCUSSION}

\section{Fatty Acid Biohydrogenation In Vitro}

Numerous in vitro and in vivo studies have enabled the major pathways of ruminal LeA biohydrogenation to be elucidated (Harfoot and Hazlewood, 1988). More recent investigations have provided further evidence on the formation of minor intermediates during the biohydrogenation of LeA in vitro (Jouany et al., 2007; Or-Rashid et al., 2011), mechanisms responsible for the formation of CLA and 18:1 in the rumen (Wallace et al., 2007; McKain et al., 2010), and the relative rates of these reactions (Troegeler-Meynadier et al., 2006; Ribeiro et al., 2007). However, no single experiment has provided a comprehensive evaluation of the intermediates that accumulate during incubations of LeA with strained ruminal fluid and the relative rates of their formation and reduction.

In the current experiment, a batch culture method was used to examine the fate of LeA during incubations with strained ruminal fluid over a range of doses that could be expected under physiological conditions. The amounts of added LeA varied between 20 and 200 $\mathrm{mg} / \mathrm{L}$, which is in the range of concentrations of LeA in ruminal digesta of grazing animals (Fievez et al., 2007), and equivalent to an LeA intake of between 0.2 and 2 $\mathrm{kg} / \mathrm{d}$ in lactating cows assuming a mean rumen pool size of $100 \mathrm{~kg}$ (Ahvenjärvi et al., 2006). High concentrations of LeA are known to inhibit microbial growth, depending on bacterial species, and arrest the hydrogenation of unsaturated fatty acids (Polan et al., 1964; Harfoot et al., 1973b; Maia et al., 2007). Potential inhibitory effects of LeA on microbial growth were minimized in this study by dispersing LeA in a sufficient concentration of polyoxyethylene sorbitan monooleate detergent (Tween 80) that is known to decrease the toxic effects of LeA on Propionibacterium freudenreichii ssp. shermanii JS (Rainio et al., 2001). A recent comparison also reported that emulsions containing LeA prepared using Tween 80 , rather than ethanol or sonication, results in more extensive hydrogenation of LeA, greater accumulation of 18:1 intermediates, and higher 18:0 production during incubations with rumen fluid (Khiaosa-ard et al., 

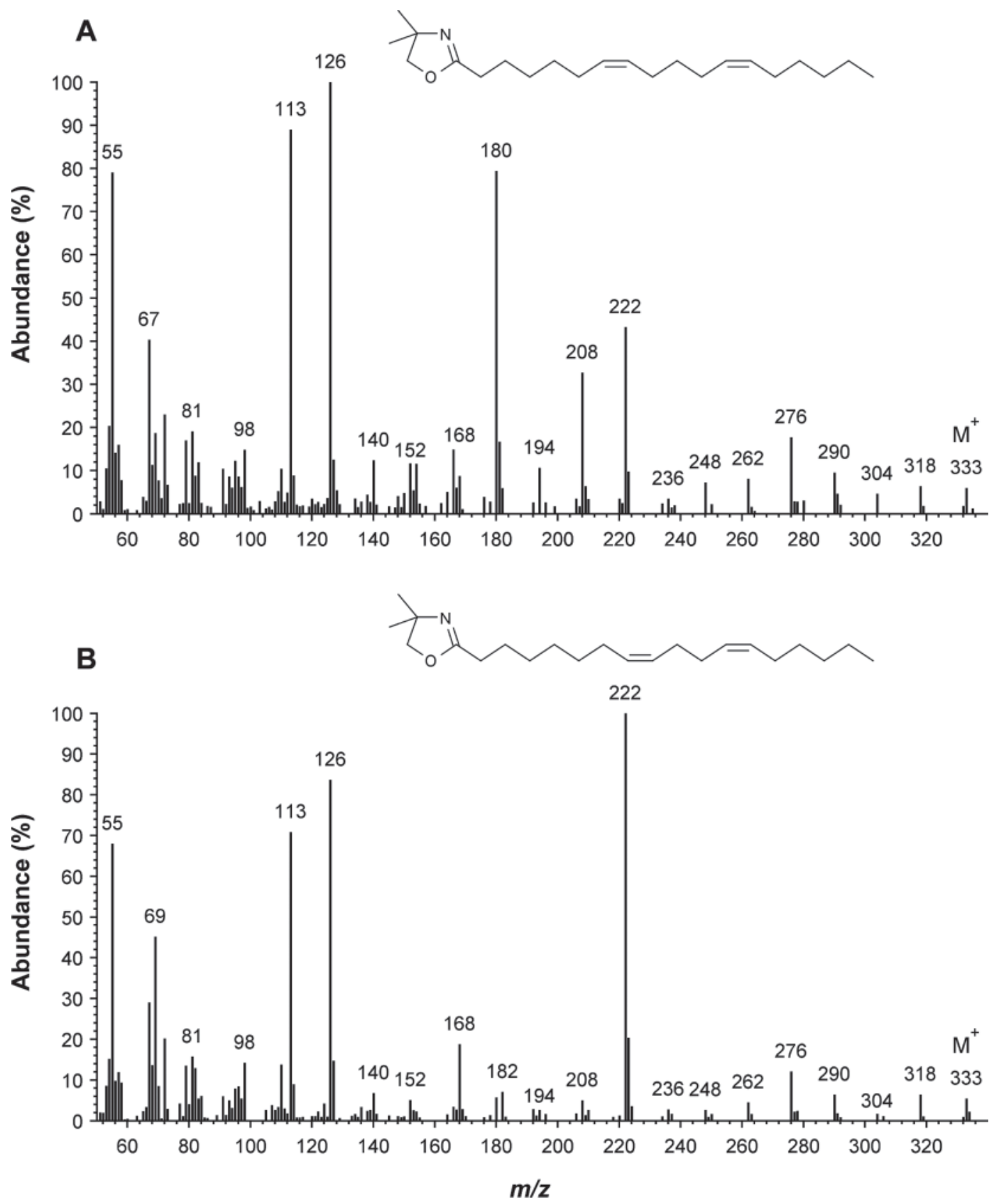

Figure 8. Mass spectrum of the 4,4-dimethyloxazoline derivative of (A) cis-7,cis-12 18:2 and (B) cis-8,cis-12 18:2, formed during incubations of linoleic acid with strained ruminal fluid.

2010), with the implication that the use of surfactant does not cause substantial interference in the biohydrogenation of LeA in vitro.

Incubations were limited up to $9 \mathrm{~h}$, to avoid potential losses in the activity of microbes in rumen fluid, which can result in the production of biohydrogenation intermediates in vitro being erroneous with respect to ruminal lipid metabolism in vivo (Jouany et al., 2007). Measurements of fatty acid flow at the duodenum com- bined with rumen evacuations have indicated that LeA is hydrogenated at a rate of between 14.6 and $16.7 \% / \mathrm{h}$ in the rumen of lactating cows (Harvatine and Allen, 2006), indicating that the duration of in vitro incubations in this experiment extend across the range of ruminal LeA turnover rates estimated in vivo.

Previous studies have demonstrated that the amount of added substrate is the major determinant of the rate and extent of LeA biohydrogenation in vitro, whereas 
Table 5. Effect of incremental addition of linoleic acid (LeA) on mean accumulation of nonconjugated octadecadienoic acids during incubations with strained ruminal fluid

\begin{tabular}{|c|c|c|c|c|c|c|c|}
\hline \multirow{2}{*}{$\begin{array}{l}\text { Amount } \\
(\mu \mathrm{g} / \text { flask })^{1}\end{array}$} & \multicolumn{4}{|c|}{ LeA addition (mg/flask) } & \multirow[b]{2}{*}{ SEM } & \multicolumn{2}{|c|}{$P$-value ${ }^{2}$} \\
\hline & 1.0 & 2.5 & 5.0 & 10.0 & & $\mathrm{~L}$ & $\mathrm{Q}$ \\
\hline cis- 6, cis- $12 \quad 18: 2^{3}$ & 8.68 & 24.8 & 41.3 & 75.0 & 8.01 & $<0.001$ & 0.706 \\
\hline cis-7,cis-12 18:2 & 7.20 & 17.3 & 60.1 & 227 & 11.95 & $<0.001$ & 0.007 \\
\hline cis-8, cis-12 18:2 & 18.5 & 28.6 & 95.4 & 338 & 19.50 & $<0.001$ & 0.009 \\
\hline cis-9,trans-12 18:2 & 11.7 & 11.8 & 19.0 & 22.0 & 2.39 & 0.004 & 0.481 \\
\hline trans- 8, cis- $12 \quad 18: 2+\Delta 4,12 \quad 18: 2$ & 20.2 & 12.4 & 20.1 & 73.1 & 9.43 & $<0.001$ & 0.054 \\
\hline trans-9, cis-12 18:2 & 0.00 & 7.29 & 35.5 & 108 & 6.14 & $<0.001$ & 0.078 \\
\hline trans-11,cis-15 18:2 & 5.91 & 8.00 & 11.6 & 40.1 & 5.60 & $<0.001$ & 0.145 \\
\hline trans -9, trans $-12 \quad 18: 2$ & 6.91 & 5.66 & 8.46 & 36.6 & 3.550 & $<0.001$ & 0.034 \\
\hline
\end{tabular}

${ }^{1}$ Values represent least squares means measured as a difference relative to the control determined after 0-, 1.5-, $3.0-, 4.5-, 6.0-$, and 9.0-h incubations $(\mathrm{n}=5)$.

${ }^{2}$ Significance of linear (L) and quadratic (Q) components of the response to LeA addition. Cubic responses to the amount of LeA incubated were not significant $(P>0.05)$.

${ }^{3}$ Contains cis-5,cis-12 18:2 as a minor component.

the composition of diet or the amount of lipid supplements fed to donor animals may also influence the disappearance of added LeA during incubations with rumen fluid (Beam et al., 2000; Fievez et al., 2007).
In the current experiment, rumen fluid was sampled from the dorsal sac at the same time each day from a nonlactating cow fed a diet containing relatively low amounts of lipid, and incubations were completed over
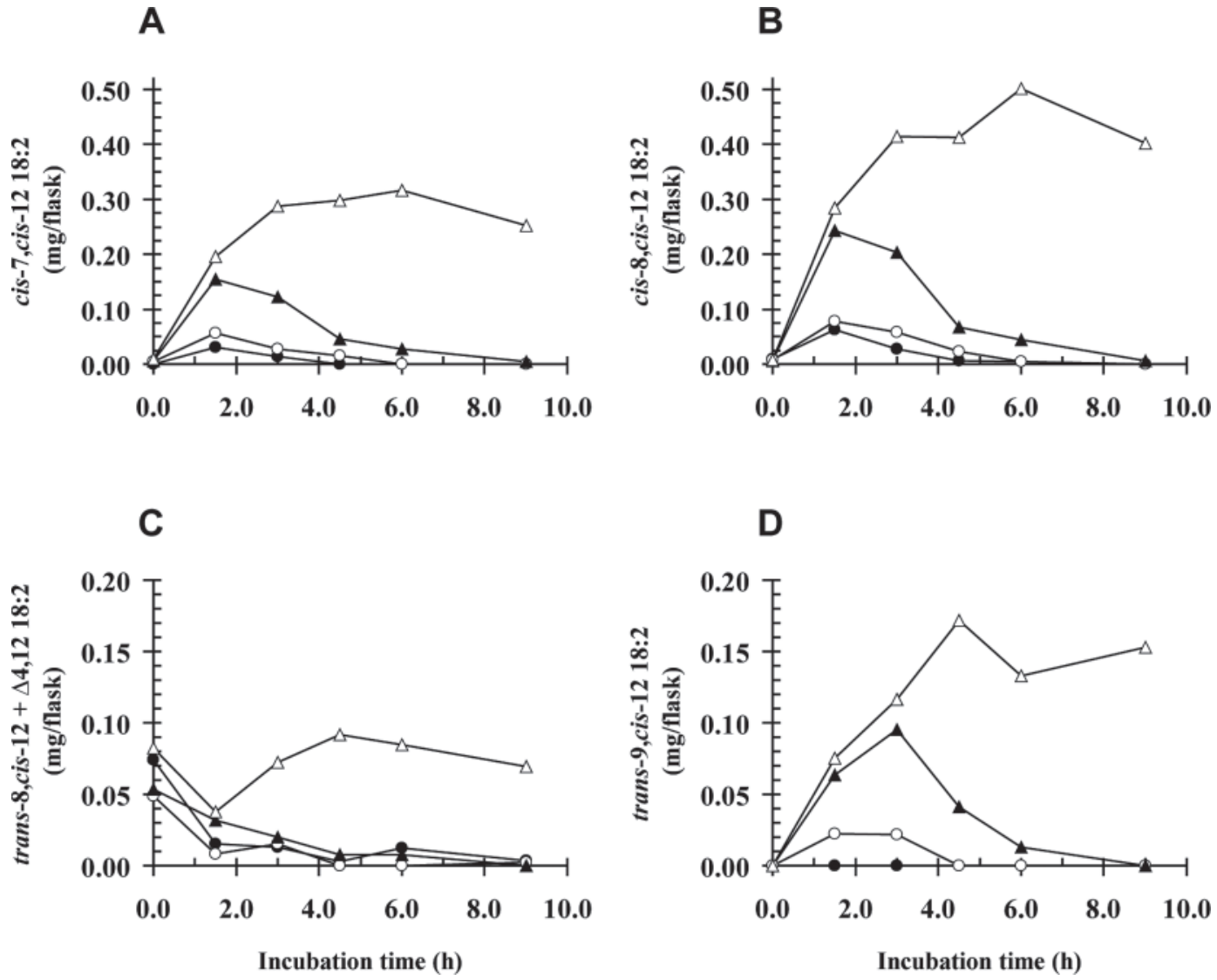

Figure 9. Temporal changes in the accumulation of (A) cis-7,cis-12 18:2; (B) cis-8,cis-12 18:2; (C) unresolved trans-8, cis-12 and 4,12 18:2; and (D) trans-9,cis-12 18:2 during incubations of $1.0(\boldsymbol{\bullet}), 2.5(\mathrm{O}), 5.0(\mathbf{\Delta})$, and $10.0(\Delta) \mathrm{mg}$ of linoleic acid with strained ruminal fluid. Values represent least squares means $(\mathrm{n}=5 ; \mathrm{SEM}=0.033,0.052,0.027$, and $0.017 \mathrm{mg} /$ flask for cis-7,cis-12 18:2; cis-8,cis-12 18:2; trans-8,cis-12 + 4,12 18:2; and trans-9,cis-12 18:2, respectively). 
Table 6. Effect of incremental addition of linoleic acid (LeA) on mean accumulation of octadecenoic acids during incubations with strained ruminal fluid

\begin{tabular}{|c|c|c|c|c|c|c|c|}
\hline \multirow{2}{*}{$\begin{array}{l}\text { Amount } \\
(\mu \mathrm{g} / \text { flask })^{1}\end{array}$} & \multicolumn{4}{|c|}{ LeA addition (mg/flask) } & \multirow[b]{2}{*}{ SEM } & \multicolumn{2}{|c|}{$P$-value ${ }^{2}$} \\
\hline & 1.0 & 2.5 & 5.0 & 10.0 & & $\mathrm{~L}$ & $\mathrm{Q}$ \\
\hline cis-9 18:1 & 39.4 & 124 & 273 & 1,070 & 39.7 & $<0.001$ & $<0.001$ \\
\hline cis-11 18:1 & 11.5 & 14.2 & 23.9 & 59.3 & 5.30 & $<0.001$ & 0.072 \\
\hline cis-12 18:1 & 78.9 & 219 & 488 & 828 & 61.2 & $<0.001$ & 0.353 \\
\hline trans- $6,-7,-8$ 18:1 & 10.4 & 28.4 & 75.9 & 92.0 & 12.14 & $<0.001$ & 0.053 \\
\hline trans-9 18:1 & 6.42 & 23.6 & 59.9 & 99.2 & 8.31 & $<0.001$ & 0.197 \\
\hline trans-10 18:1 & 23.0 & 65.9 & 223 & 475 & 24.1 & $<0.001$ & 0.785 \\
\hline trans-11 18:1 & 40.8 & 96.6 & 220 & 530 & 32.5 & $<0.001$ & 0.321 \\
\hline trans-12 18:1 & 12.9 & 41.7 & 99.3 & 98.2 & 17.94 & $<0.001$ & 0.018 \\
\hline trans-13, -14 18:1 & 35.3 & 104 & 209 & 176 & 32.3 & 0.002 & 0.003 \\
\hline trans-15 18:1 & 17.9 & 43.5 & 82.3 & 65.9 & 10.22 & 0.002 & 0.001 \\
\hline trans-16 18:1 & 17.9 & 37.7 & 64.5 & 27.0 & 8.56 & 0.622 & $<0.001$ \\
\hline
\end{tabular}

${ }^{1}$ Values represent least squares means measured as a difference relative to the control determined after 0-, 1.5-, 3.0-, 4.5-, 6.0-, and 9.0-h incubations $(\mathrm{n}=5)$.

${ }^{2}$ Significance of linear (L) and quadratic (Q) components of the response to LeA addition. Cubic responses to the amount of LeA incubated were not significant $(P>0.05)$.

a $10-d$ period to minimize between-day variations in the composition of rumen fluid. Because the adsorption onto feed particles is considered the major site of dietary unsaturated fatty acid hydrogenation by bacteria in the rumen (Harfoot et al., 1973a), LeA was added directly on top of the ground hay before the addition of rumen fluid to mimic conditions in vivo.

\section{Formation of CLA}

Incubations of incremental amounts of LeA with strained rumen fluid over periods from 0 to $9 \mathrm{~h}$ confirmed that one of the pathways of LeA biohydrogenation involved the formation of cis-9,trans-11 CLA and subsequent reduction to trans-11 18:1 and 18:0, consistent with the findings of much earlier studies (Harfoot and Hazlewood, 1988). However, several geometric isomers of $\Delta 9,11$ and $\Delta 10,12$ CLA were found to accumulate, with trans-10, cis-12 CLA being the other major conjugated intermediate, consistent with previous investigations of LeA biohydrogenation by mixed ruminal microbiota or pure strains of ruminal bacteria (Jouany et al., 2007; Maia et al., 2007; Wallace et al., 2007) and ruminal protozoa (Or-Rashid et al., 2011). Incubations of LeA also resulted in the accumulation of other isomers of CLA, consistent with previous studies in vitro (Jouany et al., 2007; Wallace et al., 2007; OrRashid et al., 2011). Furthermore, inclusion of plant oils rich in LeA have also been shown to increase 1 or more of these CLA isomers in ruminal (Loor et al., 2002) or omasal (Shingfield et al., 2008) digesta in lactating cows.

Classical studies of fatty acid metabolism by rumen bacteria have considered that the first committed step of LeA hydrogenation proceeds via the isomerization of the cis-12 double bond (Harfoot and Hazlewood, 1988). More recent studies have provided evidence that hydrogenation of LeA by rumen bacteria occurs via 2 distinct mechanisms, one involving direct isomerization, yielding geometric isomers of $\Delta 10,12 \mathrm{CLA}$, the other resulting in the formation of geometric $\Delta 9,11$ CLA isomers, possibly mediated by a hydrogen-abstraction catalyzed reaction (Wallace et al., 2007). The same mechanisms also appear to account for the formation of $\Delta 9,11$ CLA and $\Delta 10,12$ CLA isomers during incubations of LeA with bacteria isolated from the human intestine (McIntosh et al., 2009). An additional mechanism for the conversion of LeA to cis-9,trans-11 CLA by certain human gut-colonizing bacteria has been suggested to involve sequential hydration and dehydration reactions via the formation of 10-OH, cis-12 18:1 (Devillard et al., 2007). In this experiment, formation of cis-9,trans-11 CLA was instantaneous following the addition of LeA to the fermentation flask and was rapidly hydrogenated, whereas trans-10, cis-12 CLA tended to accumulate, reaching a plateau after $1.5 \mathrm{~h}$ of incubation. Appearance of cis-9, trans-11 CLA during incubations of LeA with rumen fluid is known to be transient and much lower relative to other intermediates and end products (Fellner et al., 1997; Loor et al., 2002). Under certain conditions, cis-9,trans-11 CLA and trans-10,cis-12 CLA have been reported to accumulate during incubations of LeA with mixed rumen microbes (Wąsowska et al., 2006; Wallace et al., 2007; Or-Rashid et al., 2011). Differences with respect to the accumulation of CLA isomers in this and earlier studies appear to be related to both the methods used to introduce LeA into incubation vessels and the amount of added substrate. Flask contents were devoid of trans-9,cis-11 CLA during incubations with LeA in this experiment. Earlier reports have shown that the 
A

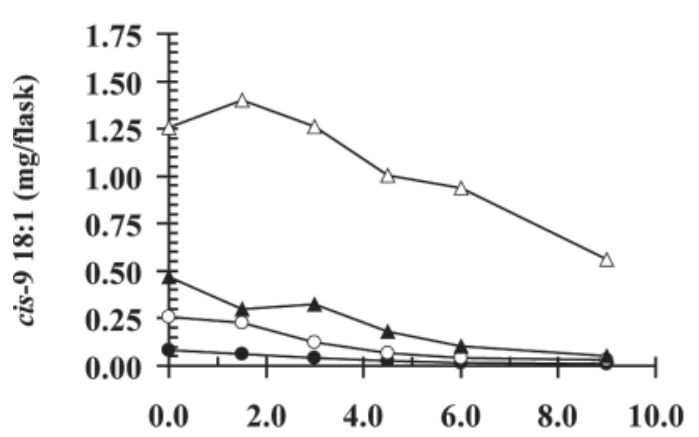

C

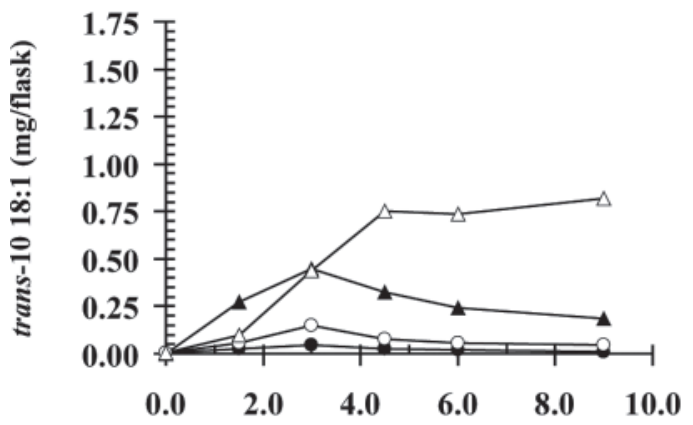

E

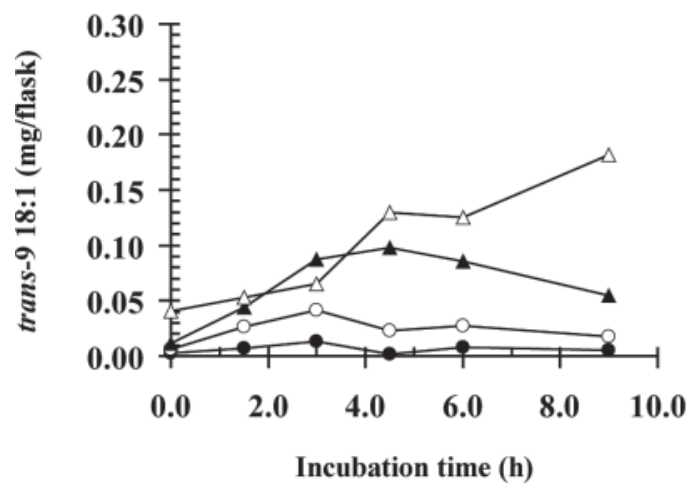

B

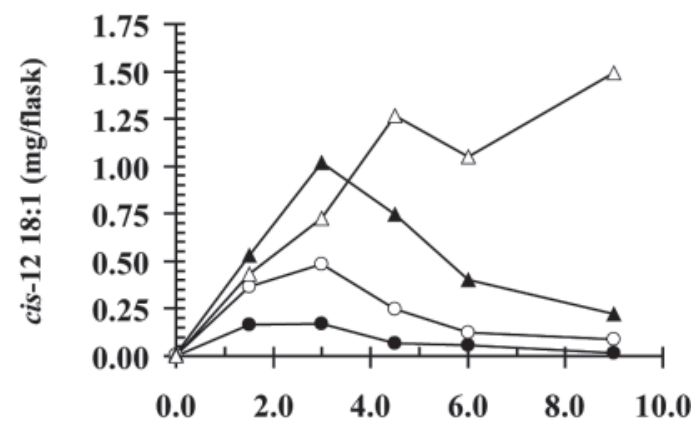

D

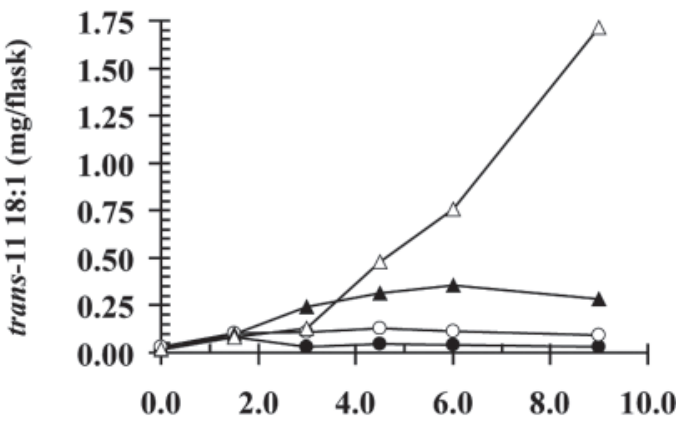

F

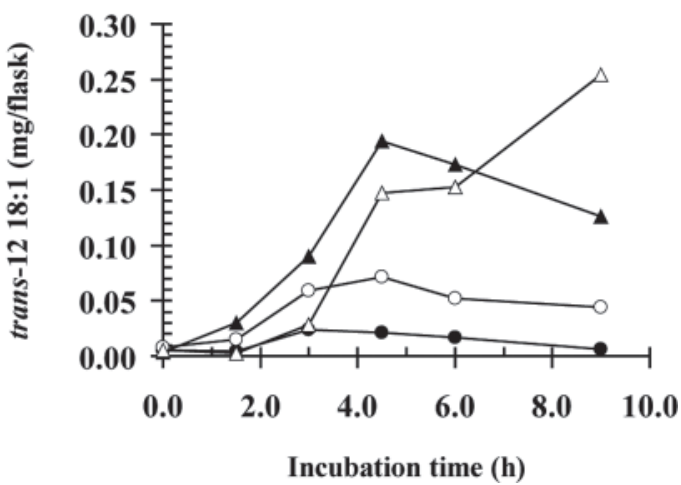

Figure 10. Temporal changes in the accumulation of (A) cis-9 18:1; (B) cis-12 18:1; (C) trans-10 18:1; (D) trans-11 18:1; (E) trans-9 18:1; and (F) trans-12 18:1 during incubations of $1.0(\bullet), 2.5(\bigcirc), 5.0(\mathbf{\Delta})$, and $10.0(\Delta) \mathrm{mg}$ of linoleic acid with strained ruminal fluid. Values represent least squares means $(\mathrm{n}=5 ; \mathrm{SEM}=0.080,0.17,0.020,0.069,0.091$, and $0.042 \mathrm{mg} /$ flask for cis-9 18:1, cis-12 18:1, trans-9 18:1, trans-10 18:1, trans-11 18:1, and trans-12 18:1, respectively).

addition of LA (50-500 $\mathrm{mg} / \mathrm{L})$ result in minor amounts of trans-9,cis-11 CLA being formed during incubations with mixed ovine ruminal microbes, Butyrivibrio fibrisolvens and Clostridium proteoclasticum P-18 (Wallace et al., 2007), and mixed bovine ruminal bacteria and protozoa (Or-Rashid et al., 2011). In contrast, no trans-9,cis-11 CLA was reported to be formed during incubations of $1-{ }^{13} \mathrm{C}$ LeA with mixed rumen microbes (Jenkins et al., 2008).
Fermentation flasks were also found to contain small amounts of trans-7,cis-9 CLA that were independent of the amount of added LeA. In contrast, analysis of lipid extracted from ruminal fluid (Corl et al., 2002), duodenal (Piperova et al., 2002), or omasal (Shingfield et al., 2003) digesta in cattle has been shown to be devoid of trans-7,cis-9 CLA. The reasons for the differences between in vitro and in vivo reports are not obvious, but there was no indication from this investigation that 
trans-7, cis-9 CLA is an intermediate of LeA biohydrogenation.

\section{Formation of Nonconjugated Octadecadienoic Intermediates}

Incubations of LeA with ruminal fluid have been reported to result in the accumulation of cis-9,trans-12 18:2; trans-9,cis-12 18:2; and trans-9,trans-12 18:2 isomers (Loor et al., 2002; Jouany et al., 2007). During incubations of LeA with strained ruminal fluid in this experiment, several unusual 18:2 intermediates, identified as $\Delta 4,1218: 2$; cis-5,cis-12 18:2; cis-6,cis-12 18:2; cis-7,cis-12 18:2; cis-8,cis-12 18:2; and trans-8,cis-12 18:2, were also found to accumulate.

Prior to the analysis of flask contents, the methods used for lipid extraction and methylation of NEFA were evaluated extensively using authentic FAME and NEFA standards. Based on these investigations, no indications were found that these procedures caused isomerization, hydration, or oxidization of unsaturated fatty acids, including LeA. Therefore, the appearance and accumulation of unusual cis, cis octadecadienoic acids, at least under the specified conditions of this experiment, occur during the hydrogenation of LeA. No reports have been made of $\Delta 4,1218: 2$; cis-5, cis-12 18:2; cis-6,cis-12 18:2; cis-7,cis-12 18:2; or cis-8,cis-12 18:2 concentrations in ruminal digesta, but cis-6,cis-10 18:2 has been detected in butter oil (Nikolova et al., 2006).

The appearance of minor 18:2 intermediates containing a cis-12 double bond also provides evidence that biohydrogenation of LeA also proceeds by a mechanism other than hydrogen abstraction or isomerization of the cis-12 double bond (Wallace et al., 2007; McIntosh et al., 2009). Several NC 18:2 isomers, including cis8,cis-12 18:2; cis-8,trans-12 18:2; cis-9,cis-13 18:2; and cis-9,trans-13 18:2, are formed during hydrogenation of LeA with iridium, palladium, and ruthenium catalysts as a result of the release of the hydrogen atom from the adjacent methylene group at $\Delta 8$ or $\Delta 14$ of the semihydrogenated $\mathrm{C}-\mathrm{C}$ bond and rotation of the $\mathrm{C}-\mathrm{C}$ bond during abstraction of the hydrogen atom (Kitayama et al., 1997). Further investigations are required to elucidate mechanisms responsible for the formation of minor intermediates during the hydrogenation of LeA with strained ruminal fluid.

\section{Formation of Octadecenoic Intermediates}

Incremental addition of LeA resulted in the accumulation of several 18:1 intermediates in fermentation flasks with cis-9 18:1, cis-12 18:1, trans-10 18:1, and trans-11 18:1 as the main products, consistent with previous ob- servations (Harfoot and Hazlewood, 1988; Enjalbert et al., 2003; Jouany et al., 2007). The appearance of cis-9 18:1 and cis-12 18:1 formed during incubations with ruminal fluid could be considered as evidence of LeA biohydrogenation involving the direct reduction of $\mathrm{cis}-9$ and cis-12 double bonds, but it is also possible that these 18:1 intermediates originate from the hydrogenation of 18:2 intermediates. During incubations of LeA with strained ruminal fluid, trans-10,cis-12 CLA was found to accumulate before that of cis-12 18:1, trans-10 18:1, and trans-12 18:1. These findings are consistent with reports that pure cultures of ruminal Butyrivibrio fibrisolvens hydrogenate a mixture of trans-10,cis-12 CLA and trans-10,trans-12 CLA to cis-12 18:1, trans-10 18:1, and trans-12 18:1 (McKain et al., 2010), and ruminal infusions of a mixture of CLA isomers containing trans-10, cis-12 CLA result in a dose-dependent increase in plasma and milk fat trans-10 18:1 concentrations in lactating cows (Loor and Herbein, 2001).

Both trans-10 18:1 and trans-11 18:1 were found to accumulate, but the addition of greater amounts of LeA resulted in a linear increase in the ratio of trans-10 18:1 to trans-11 18:1. In earlier studies, the ratio of trans-10 18:1 to trans-11 18:1 has been shown to increase 2 -fold over $12 \mathrm{~h}$ during incubations of LeA with rumen bacteria but remain unchanged with rumen protozoa or mixed microbes (Or-Rashid et al., 2011). Changes in the formation and accumulation of these trans 18:1 intermediates have been reported in cows fed high-concentrate diets or low-forage diets containing oils rich in polyunsaturated fatty acids (Griinari et al. 1998; Piperova et al., 2002; Loor et al., 2004), with evidence that alterations in ruminal biohydrogenation under these conditions occur over time (Roy et al., 2006; Shingfield et al., 2006).

Under the controlled conditions of this experiment, the shift toward trans-10 at the expense of trans-11 18:1 occurred over a relatively short incubation period. Previous reports have indicated that decreases in $\mathrm{pH}$ promote the formation of trans-10,cis-12 CLA and trans-10 18:1 from LeA by ruminal microbes in vitro (TroegelerMeynadier et al., 2003; Fuentes et al., 2009). However, the mean decrease in $\mathrm{pH}$ over the course of incubations in this experiment was marginal (0.4 unit) and independent of the amount of LeA added. Therefore, the changes in the ratio of trans-10 18:1 to trans-11 18:1 in the present investigation are not readily explained by differences in rates of $\Delta 9,11$ CLA and $\Delta 10,12$ CLA formation but may reflect time-dependent changes in the relative rates in the reduction of 18:2 intermediates. Recent studies examining the products formed during hydrogenation of CLA isomers by Butyrivibrio fibrisolvens suggest that the reduction of geometric iso- 
mers of $\Delta 9,11$ CLA occurs via a different mechanism responsible for the hydrogenation of other 18-carbon unsaturated fatty acids (McKain et al., 2010).

Incubations with strains of Enterococcus fecalis isolated from the rumen indicated that LeA may also be hydrated to yield $10-\mathrm{OH}$, cis-12 18:1, and cis-9, $13-\mathrm{OH}$ 18:1 (Hudson et al., 1998), whereas the reduction of cis-9 18:1 and trans-11 18:1 by Propionibacterium acnes involves the formation of 10-O-18:0 via an 10-OH-18:0 intermediate (McKain et al., 2010). Analysis of FAME by GC did not permit the quantification of oxygenated 18-carbon fatty acids in this experiment. However, the amount of 18-carbon fatty acids in fermentation flasks $(1.15,2.50,4.97$, and $10.08 \mathrm{mg} /$ flask) following the addition of $1.0,2.5,5.0$, and $10.0 \mathrm{mg}$ of LeA, respectively, indicates that hydration did not represent a major route of LeA transformation in this study.

\section{Reduction of Octadecenoic Intermediates to Stearic Acid}

Conversion of trans 18:1 to 18:0 is known to be inhibited by high LeA concentrations, an effect attributed to irreversible substrate (Kepler and Tove, 1967) or endproduct inhibition (Polan et al., 1964; Harfoot et al., 1973b; Beam et al., 2000) of octadecenoic acid reductase activity, or a combination of both mechanisms. Recent studies have shown that the growth of Clostridium proteoclasticum $\mathrm{P}-18$, a ruminal bacterium capable of producing 18:0, can be inhibited during incubations with trans 18:1 and demonstrated that the more unsaturated fatty acids exert more toxic effects on the ruminal bacteria involved in biohydrogenation (Maia et al., 2007). However, the rates of disappearance and appearance of CLA isomers during incubations with $10 \mathrm{mg}$ of LeA in the present study were constant, indicating that neither a decrease in substrate concentrations nor the accumulation of trans and cis intermediates altered the initial rate of LeA biohydrogenation with the implication that the rate of the initial isomerization of LeA is finite, consistent with previous considerations (TroegelerMeynadier et al., 2006). Furthermore, incubations of 5 and $10 \mathrm{mg}$ of LeA with strained ruminal fluid caused NC 18:2 and 18:1 intermediates to accumulate over time, which could be interpreted as evidence that $\mathrm{NC}$ 18:2 isomers inhibit the reduction of 18:1 to 18:0.

\section{Kinetics of LeA Biohydrogenation}

A multiple-pool, first-order model (Ribeiro et al., 2007) was used to estimate the rates of transfer from various fatty acid pools to provide further insight into the kinetics of LeA biohydrogenation. Modeling of the appearance of intermediates during incubations with 5.0 and $10.0 \mathrm{mg}$ of LeA indicated that the rates of transfer of LeA to the CLA pool were almost identical to the transfer from the LeA to NC 18:2 pool, suggesting that these reactions, which involve different mechanisms, may occur at the same rate. However, the CLA pool was, in the main, comprised of trans-10,cis-12 CLA, whereas the conversion of LeA to cis-9,trans-11 CLA was found to occur almost instantaneously. It would, therefore, appear that the kinetics of LeA isomerization to 10,12 geometric isomers of CLA are similar to the rates of LeA conversion to NC 18:2.

Transfer rates from the CLA pool to $18: 1$ pool were higher compared with the rate of transfer from the $\mathrm{NC}$ 18:2 to 18:1 pool. However, estimates of transfer of LeA to CLA and from CLA to $18: 1$ pools have to be interpreted with caution, and considered more apparent than true, because of the limited accumulation of CLA isomers over time and instantaneous formation of $\Delta 9,11$ CLA and $\Delta 10,12$ CLA immediately after the addition of LeA to incubation flasks. As a result, the rate of CLA isomer reduction in this experiment was 7.3 and $19.1 \% / \mathrm{h}$, being lower than values of 23.5 to $27.4 \% / \mathrm{h}$, when the production of CLA did not account for LeA being converted to $\mathrm{NC}$ 18:2 intermediates (Ribeiro et al., 2007). Transfer of LeA to the NC 18:2 isomer pool was estimated to vary between 7.3 and $18.1 \% / \mathrm{h}$, depending on the amount of added LeA, which would account for the differences in the initial rates of LeA isomerization between the present and earlier experiments.

Estimates of transfer from the 18:1 pool to 18:0 were much lower compared with fractional rates for other steps of LeA biohydrogenation and decreased in direct relation to the amount of LeA incubated, in agreement with earlier reports (Troegeler-Meynadier et al., 2003; Ribeiro et al., 2007). Overall, the use of a dynamic model as well as considerations of temporal changes in intermediates formed during incubations of LeA with strained ruminal fluid indicated that the final reduction of 18:1 intermediates as rate limiting, and suggest that the reduction of CLA isomers occurs at a faster rate than the reduction of $\mathrm{NC}$ 18:2 intermediates.

\section{CONCLUSIONS}

Disappearance of LeA during incubations with strained rumen fluid was dependent on the amount of the added substrate. Current data indicated that LeA biohydrogenation may proceed via the formation of cis-9,trans-11 CLA and serial reduction to trans-11 18:1 and 18:0, but other metabolic pathways are also involved. Biohydrogenation of LeA also resulted in the formation of other geometric isomers of $\Delta 9,11$ CLA and $\Delta 10,12 \mathrm{CLA}$, and caused cis and trans isomers of 
$\Delta 9,10,11$, and 12 18:1 to accumulate. Several intermediates, including cis-5,cis-12 18:2; cis-7,cis-12 18:2; and cis-8,cis-12 18:2 were also formed, providing evidence that the biohydrogenation of LeA may also proceed via mechanisms other than hydrogen abstraction or isomerization of the cis-12 double bond. Modeling the kinetics of LeA biohydrogenation indicated that reduction of 18:1 intermediates is rate limiting, and that the reduction of CLA isomers to 18:1 intermediates occurs at a faster rate than the reduction of $\mathrm{NC}$ 18:2 intermediates.

\section{ACKNOWLEDGMENTS}

The authors gratefully acknowledge and appreciate the assistance of Minna Aalto, Piia Kairenius, and Laura Ventto (MTT Agrifood Research Finland) during lipid analysis.

\section{REFERENCES}

Ahvenjärvi, S., E. Joki-Tokola, A. Vanhatalo, S. Jaakkola, and P. Huhtanen. 2006. Effects of replacing grass silage with barley silage in dairy cow diets. J. Dairy Sci. 89:1678-1687.

Beam, T. M., T. C. Jenkins, P. J. Moate, R. A. Kohn, and D. L. Palmquist. 2000. Effects of amount and source of fat on the rates of lipolysis and biohydrogenation of fatty acids in ruminal contents. J. Dairy Sci. 83:2564-2573.

Christie, W. W. 1998. Gas chromatography-mass spectrometry methods for structural analysis of fatty acids. Lipids 33:343-353.

Corl, B. A., L. H. Baumgard, J. M. Griinari, P. Delmonte, K. M. Morehouse, M. P. Yurawecz, and D. E. Bauman. 2002. Trans7,cis-9 CLA is synthesized endogenously by $\Delta^{9}$-desaturase in dairy cows. Lipids 37:681-688.

Cruz-Hernandez, C., Z. Deng, J. Zhou, A. R. Hill, M. P. Yurawecz, P. Delmonte, M. M. Mossoba, M. E. R. Dugan, and J. K. G. Kramer. 2004. Methods for analysis of conjugated linoleic acids and trans-18:1 isomers in dairy fats by using a combination of gas chromatography, silver-ion thin-layer chromatography/gas chromatography, and silver-ion liquid chromatography. J. AOAC Int. $87: 545-562$.

Delmonte, P., A. Kataoka, B. A. Corl, D. E. Bauman, and M. P. Yurawecz. 2005. Relative retention order of all isomers of cis/trans conjugated linoleic acid FAME from the 6,8- to 13,15-positions using silver ion HPLC with two elution systems. Lipids 40:509-514.

Devillard, E., F. M. McIntosh, S. H. Duncan, and R. J. Wallace. 2007. Metabolism of linoleic acid by human gut bacteria: Different routes for biosynthesis of conjugated linoleic acid. J. Bacteriol. 189:2566-2570.

Enjalbert, F., P. Eynard, M. C. Nicot, A. Troegeler-Meynadier, C. Bayourthe, and R. Moncoulon. 2003. In vitro versus in situ ruminal biohydrogenation of unsaturated fatty acids from a raw or extruded mixture of ground canola seed/canola meal. J. Dairy Sci. 86:351-359.

Fay, L., and U. Richli. 1991. Location of double bonds in polyunsaturated fatty acids by gas chromatography-mass spectrometry after 4,4-dimethyloxazoline derivatization. J. Chromatogr. A 541:8998.

Fellner, V., F. D. Sauer, and J. K. G. Kramer. 1997. Effect of nigericin, monensin, and tetronasin on biohydrogenation in continuous flowthrough ruminal fermenters. J. Dairy Sci. 80:921-928.

Fievez, V., B. Vlaeminck, T. Jenkins, F. Enjalbert, and M. Doreau. 2007. Assessing rumen biohydrogenation and its manipulation in vivo, in vitro and in situ. Eur. J. Lipid Sci. Technol. 109:740-756.

Fuentes, M. C., S. Calsamiglia, P. W. Cardozo, and B. Vlaeminck. 2009. Effect of $\mathrm{pH}$ and level of concentrate in the diet on the pro- duction of biohydrogenation intermediates in a dual-flow continuous culture. J. Dairy Sci. 92:4456-4466.

Griinari, J. M., D. A. Dwyer, M. A. McGuire, D. E. Bauman, D. L. Palmquist, and K. V. V. Nurmela. 1998. Trans-octadecenoic acids and milk fat depression in lactating cows. J. Dairy Sci. 81:1251-1261.

Harfoot, C. G., and G. P. Hazlewood. 1988. Lipid metabolism in the rumen. Pages 285-322 in The Rumen Microbial Ecosystem. Elsevier Science Publishing, New York, NY.

Harfoot, C. G., R. C. Noble, and J. H. Moore. 1973a. Food particles as a site for biohydrogenation of unsaturated acids in the rumen. Biochem. J. 132:829-832.

Harfoot, C. G., R. C. Noble, and J. H. Moore. 1973b. Factors influencing the extent of biohydrogenation of linoleic acid by rumen microorganisms in vitro. J. Sci. Food Agric. 24:961-970.

Harvatine, K. J., and M. S. Allen. 2006. Fat supplements affect fractional rates of ruminal fatty acid biohydrogenation and passage in dairy cows. J. Nutr. 136:677-685.

Hudson, J. A., B. Morvan, and K. N. Joblin. 1998. Hydration of linoleic acid by bacteria isolated from ruminants. FEMS Microbiol. Lett. 169:277-282.

Jenkins, T. C., R. J. Wallace, P. J. Moate, and E. E. Mosley. 2008. Board-invited review: Recent advances in biohydrogenation of unsaturated fatty acids within the rumen microbial ecosystem. J. Anim. Sci. 86:397-412.

Jouany, J.-P., B. Lassalas, M. Doreau, and F. Glasser. 2007. Dynamic features of the rumen metabolism of linoleic acid, linolenic acid and linseed oil measured in vitro. Lipids 42:351-360.

Kawashima, H., and M. Ohnishi. 2004. Identification of minor fatty acids and various nonmethylene-interrupted diene isomers in mantle, muscle, and viscera of the marine bivalve Megangulus zyonoensis. Lipids 39:265-271.

Kepler, C. R., and S. B. Tove. 1967. Biohydrogenation of unsaturated fatty acids III. Purification and properties of a linoleate $\Delta^{12}$-cis, $\Delta^{11}$-trans-isomerase from Butyrivibrio fibrisolvens. J. Biol. Chem. 242:5686-5692.

Khiaosa-ard, R., F. Leiber, and C. R. Soliva. 2010. Methods of emulsifying linoleic acid in biohydrogenation studies in vitro may bias the resulting fatty acid profiles. Lipids 45:651-657.

Kitayama, Y., M. Muraoka, M. Takahashi, T. Kodama, E. Takahashi, and M. Okamura. 1997. Catalytic hydrogenation of linoleic acid over platinum-group metals supported on alumina. J. Am. Oil Chem. Soc. 74:525-529.

Loor, J. J., A. B. P. A. Bandara, and J. H. Herbein. 2002. Characterization of 18:1 and 18:2 isomers produced during microbial biohydrogenation of unsaturated fatty acids from canola and soya bean oil in the rumen of lactating cows. J. Anim. Physiol. Anim. Nutr. (Berl.) 86:422-432.

Loor, J. J., and J. H. Herbein. 2001. Alterations in blood plasma and milk fatty acid profiles of lactating Holstein cows in response to ruminal infusion of a conjugated linoleic acid mixture. Anim. Res. 50:463-476.

Loor, J. J., K. Ueda, A. Ferlay, Y. Chilliard, and M. Doreau. 2004. Biohydrogenation, duodenal flow, and intestinal digestibility of trans fatty acids and conjugated linoleic acids in response to dietary forage concentrate ratio and linseed oil in dairy cows. J. Dairy Sci. 87:2472-2485.

Lourenço, M., E. Ramos-Morales, and R. J. Wallace. 2010. The role of microbes in rumen lipolysis and biohydrogenation and their manipulation. Animal 4:1008-1023.

Maia, M. R. G., L. C. Chaudhary, L. Figueres, and R. J. Wallace. 2007. Metabolism of polyunsaturated fatty acids and their toxicity to the microflora of the rumen. Antonie van Leeuwenhoek 91:303-314.

McIntosh, F. M., K. J. Shingfield, E. Devillard, W. R. Russell, and R. J. Wallace. 2009. Mechanism of conjugated linoleic acid and vaccenic acid formation in human faecal suspensions and pure cultures of intestinal bacteria. Microbiology 155:285-294.

McKain, N., K. J. Shingfield, and R. J. Wallace. 2010. Metabolism of conjugated linoleic acids and 18:1 fatty acids by ruminal bacteria: Products and mechanisms. Microbiology 156:579-588. 
Mosley, E. E., G. L. Powell, M. B. Riley, and T. C. Jenkins. 2002 Microbial biohydrogenation of oleic acid to trans isomers in vitro. J. Lipid Res. 43:290-296.

Nikolova, D., D. Antonova, I. Marekov, and B. Nikolova-Damyanova. 2006. Bis-methylene-interrupted octadecadienoic fatty acids in Bulgarian bovine butter fats. Eur. J. Lipid Sci. Technol. 108:212217.

Or-Rashid, M. M., O. AlZahal, and B. W. McBride. 2011. Comparative studies on the metabolism of linoleic acid by rumen bacteria, protozoa, and their mixture in vitro. Appl. Microbiol. Biotechnol. 89:387-395.

Ørskov, E. R., and I. McDonald. 1979. The estimation of protein degradability in the rumen from incubation measurements weighted according to rate of passage. J. Agric. Sci. 92:499-503.

Piperova, L. S., J. Sampugna, B. B. Teter, K. F. Kalscheur, M. P. Yurawecz, Y. Ku, K. M. Morehouse, and R. A. Erdman. 2002. Duodenal and milk trans octadecenoic acid and conjugated linoleic acid (CLA) isomers indicate that postabsorptive synthesis is the predominant source of cis-9-containing CLA in lactating dairy cows. J. Nutr. 132:1235-1241.

Polan, C. E., J. J. McNeill, and C. B. Tove. 1964. Biohydrogenation of unsaturated fatty acids by rumen bacteria. J. Bacteriol. 88:1056-1064

Proell, J. M., E. E. Mosley, G. L. Powell, and T. C. Jenkins. 2002 Isomerization of stable isotopically labeled elaidic acid to cis and trans monoenes by ruminal microbes. J. Lipid Res. 43:2072-2076.

Rainio, A., M. Vahvaselkä, T. Suomalainen, and S. Laakso. 2001. Reduction of linoleic acid inhibition in production of conjugated linoleic acid by Propionibacterium freudenreichii ssp. shermanii. Can. J. Microbiol. 47:735-740.

Ribeiro, C. V., M. L. Eastridge, J. L. Firkins, N. R. St-Pierre, and D. L. Palmquist. 2007. Kinetics of fatty acid biohydrogenation in vitro. J. Dairy Sci. 90:1405-1416.

Roy, A., A. Ferlay, and Y. Chilliard. 2006. Production of butter fat rich in trans10-C18:1 for use in biomedical studies in rodents. Reprod. Nutr. Dev. 46:211-218.
Shingfield, K. J., S. Ahvenjärvi, V. Toivonen, A. Ärölä, K. V. V. Nurmela, P. Huhtanen, and J. M. Griinari. 2003. Effect of fish oil on biohydrogenation of fatty acids and milk fatty acid content in cows. Anim. Sci. 77:165-179.

Shingfield, K. J., S. Ahvenjärvi, V. Toivonen, A. Vanhatalo, P. Huhtanen, and J. M. Griinari. 2008. Effect of incremental levels of sunflower-seed oil in the diet on ruminal lipid metabolism in lactating cows. Br. J. Nutr. 99:971-983.

Shingfield, K. J., C. K. Reynolds, G. Hervás, J. M. Griinari, A. S. Grandison, and D. E. Beever. 2006. Examination of the persistency of milk fatty acid composition responses to fish oil and sunflower oil in the diet of dairy cows. J. Dairy Sci. 89:714-732.

Spitzer, V. 1996. Structure analysis of fatty acids by gas chromatography-low resolution electron impact mass spectrometry of their 4, 4-dimethyloxazoline derivatives-A review. Prog. Lipid Res. $35: 387-408$.

Troegeler-Meynadier, A., L. Bret-Bennis, and F. Enjalbert. 2006. Rates and efficiencies of reactions of ruminal biohydrogenation of linoleic acid according to $\mathrm{pH}$ and polyunsaturated fatty acids concentrations. Reprod. Nutr. Dev, 46:713-724.

Troegeler-Meynadier, A., M. C. Nicot, C. Bayourthe, R. Moncoulon, and F. Enjalbert. 2003. Effects of $\mathrm{pH}$ and concentrations of linoleic and linolenic acids on extent and intermediates of ruminal biohydrogenation in vitro. J. Dairy Sci. 86:4054-4063.

Wallace, R. J., N. McKain, K. J. Shingfield, and E. Devillard. 2007. Isomers of conjugated linoleic acids are synthesized via different mechanisms in ruminal digesta and bacteria. J. Lipid Res. 48:2247-2254.

Wąsowska, I., M. R. G. Maia, K. M. Niedźwiedzka, M. Czauderna, J. M. C. Ramalho Ribeiro, E. Devillard, K. J. Shingfield, and R. J. Wallace. 2006. Influence of fish oil on ruminal biohydrogenation of C18 unsaturated fatty acids. Br. J. Nutr. 95:1199-1211. 